\title{
Nonlinear frequency response analysis of forced periodic operation of non-isothermal CSTR using single input modulations. Part I: Modulation of inlet concentration or flow-rate
}

\author{
Daliborka Nikolića $^{\mathrm{a}}$, Andreas Seidel-Morgenstern ${ }^{\mathrm{b}}$, Menka Petkovska ${ }^{\mathrm{c}, *}$ \\ a University of Belgrade/Institute for Chemistry, Technology and Metallurgy, Njegoševa 12, 11000 Belgrade, Serbia \\ ${ }^{\mathrm{b}}$ Otto-von-Guericke University and Max-Planck Institute for Dynamics of Complex Technical Systems, Magdeburg, Germany \\ ${ }^{\mathrm{c}}$ University of Belgrade/Faculty of Technology and Metallurgy, Department of Chemical Engineering, Karnegijeva 4, 11120 Belgrade, Serbia
}

\section{H I G H L I G H T S}

- Evaluating single input periodic operations of non-isothermal CSTR by NFR method.

- Analysis for the non-isothermal, homogeneous, simple $n$-th order reaction in a CSTR.

- Derivation of asymmetrical second order FR functions and sign analysis.

- Conditions for process improvement by modulating inlet concentration or flow-rate.

- Comparison between results obtained by NFR method and by numerical simulations.

\section{A R T I C L E I N F O}

\section{Article history:}

Received 20 January 2014

Received in revised form

30 May 2014

Accepted 5 June 2014

Available online 14 June 2014

Keywords:

Non-isothermal CSTR

Nonlinear dynamics

Mathematical modelling

Chemical reactors

Simulation

Nonlinear frequency response method

\begin{abstract}
A B S T R A C T
Periodic operations of a non-isothermal CSTR with $n$-th order reaction, subject to a single input modulation, is analysed using the nonlinear frequency response (NFR) method, introduced in our previous publications. The method is based on deriving the asymmetrical second order frequency response function (FRF) and analysing its sign. In Part I of this paper, periodic operation with modulation of the inlet concentration or flow-rate of the reaction stream is analysed. As a result, conditions regarding the reaction order, process parameters and frequency of the input modulation are identified that need to be fulfilled in order to achieve process improvement through the periodic operation compared to conventional steady state operation. The method is applied for a numerical example from literature and the results obtained by the NFR method are compared with the results of numerical simulation. Good agreement is obtained, except for imposed forcing frequencies close to the resonant frequency and high forcing amplitudes.
\end{abstract}

(c) 2014 Elsevier Ltd. All rights reserved.

\section{Introduction}

Periodic operation of different chemical engineering processes, especially chemical reactors, has been a research topic of a number of research groups in the last 50 years (Douglas and Rippin, 1966; Douglas, 1967; Horn and Lin, 1967; Renken, 1972; Bailey, 1973; Watanabe et al., 1981; Schadlich et al., 1983; Silveston 1987; Sterman and Ydstie, 1990a, 1990b, 1991; Chen and Hwang, 1994; Silveston et al. 1995; Silveston, 1998).

Periodic modulation of one or more inputs can provide better average performance compared to the optimal steady-state operation (increased conversion, improved selectivity, increased yield, increased

\footnotetext{
* Corresponding author. Tel.: + 381113303 610; fax: +381 113370387

E-mail address: menka@tmf.bg.ac.rs (M. Petkovska).
}

catalytic activity etc.). The source of possible improvement lies in the process nonlinearity. Many experimental and simulation studies verify that it is often advantageous to exploit the nonlinear behaviour of chemical reactions and to operate in a dynamic regime by periodic modulation of one or more inputs (Sterman and Ydstie, 1991).

For nonlinear systems with periodic modulation one or more inputs, the average value of the output is different from the steadystate value. Although this difference is small for mild nonlinearities, for highly nonlinear systems or those which exhibit resonance, the deviations might be very significant (Douglas, 1967).

Identification of candidate systems for process enhancement through periodic operation and estimation of the magnitude of such enhancement have occupied many researchers. More details about previously proposed criteria or techniques for evaluation of periodically operated processes can be found in Petkovska and Seidel-Morgenstern (2013). 
These previous theoretical approaches have not provided yet general methods to predict the possibility of process improvements (Petkovska and Seidel-Morgenstern, 2013). In practice, testing whether a periodic operation leads to an increased productivity as compared to the corresponding steady-state operation, is usually performed by long and tedious experimental and/or numerical work. Therefore, there is still a need for developing simple and reliable methods which would enable quantitative evaluation of the possibility of process improvements through periodic operations (Petkovska and Seidel-Morgenstern, 2013).

In our previous work, we introduced the nonlinear frequency response (NFR) method, which can give in early development stages a fast answer whether working under periodic conditions could be favourable. The NFR method, which is applicable for weakly nonlinear systems (Weiner and Spina, 1980), is based on Volterra series, generalized Fourier transform and the concept of higher order frequency response functions (FRFs) (Marković et al., 2008). The NFR method also enables approximate evaluation of the magnitude of the improvement for weakly nonlinear systems if it can be achieved by periodic operation.

Till now, the NFR method has been applied for evaluation of periodic operations of different types of reactors (continuous stirred tank reactor (CSTR), plug flow tubular reactor and dispersive flow tubular reactor) with feed concentration modulation for simple isothermal homogeneous $n$-th order reactions (Marković et al., 2008). The same type of analysis was used for periodic operation of a CSTR with a simple isothermal heterogeneous catalytic $n$-th order reaction (Petkovska et al., 2010).

In one of our previous papers, the NFR method was extended for evaluation of periodic operation with simultaneous modulation of two inputs, and tested on an isothermal CSTR in which a simple isothermal $n$-th order homogeneous reaction takes place and inlet concentration and flow-rate are modulated simultaneously (Nikolić-Paunić and Petkovska, 2013).

In this paper, for the first time the NFR method is applied for evaluation of periodically operated non-isothermal reactors, for which the temperature effects of the chemical reaction cannot be neglected. This problem has already been treated in the literature (Ritter and Douglas, 1970; Sterman and Ydstie 1990a, 1990b; Dorawala and Douglas, 1971; Silveston and Hudgins, 2004). There are several parameters which could be periodically modulated: the inlet concentration, the flow-rate, the temperature of the feed stream and the temperature of the heating/cooling fluid. Since the potential for improvement through periodic operation strongly depends on the degree of the nonlinearity of the system, it is expected that the nonisothermal CSTR, which is highly nonlinear, would offer a lot of potential for process improvement. The non-isothermal CSTR is also a good test for the NFR method, considering that the method is valid for weakly nonlinear systems.

It is well-known that a non-isothermal CSTR can in principle exhibit unstable behaviour (Douglas, 1972). It should be noticed that the NFR method is applicable only for stable systems, so stability analysis should always be performed first.

In this paper, the NFR method is applied for evaluation of periodic operation of a non-isothermal CSTR in which a simple $n$-th order homogeneous reaction takes place, when either the inlet concentration and or the flow-rate are modulated inputs. In Part II of this paper, we will analyse the periodic operation of the non-isothermal CSTR when the modulated inputs are the temperature of the inlet reaction stream or the temperature of the cooling/heating fluid.

\section{Nonlinear frequency response method for evaluating periodic processes}

Frequency response (FR) represents a quasi-stationary response of the system to a periodic (sinusoidal or co-sinusoidal) input modulation, which is achieved when the transient response becomes negligible (theoretically for infinite time) (Douglas, 1972).

FR of a linear system is a periodic function of the same shape and frequency as the input, but with different amplitude and phase from the input values. The mean value of this periodic function is equal to the steady state value. Frequency response function of a linear system is defined by the amplitude ratio and the phase difference of the output and input in the quasistationary state (Douglas, 1972).

On the other hand, FR of a nonlinear system is a complex periodic function and it cannot be represented by a single frequency response function. FR of a nonlinear system, in addition to the basic harmonic, which has the same frequency as the input modulation, also contains a non-periodic, the so called DC component, and an infinite number of higher harmonics (Douglas, 1972; Weiner and Spina, 1980; Petkovska and Seidel-Morgenstern, 2013). One approach for analysing FRs of nonlinear systems is the concept of higher order frequency response functions (FRFs) which is based on Volterra series and the generalized Fourier transform (Weiner and Spina, 1980).

The nonlinear model $\boldsymbol{G}$ of a weakly nonlinear system in the frequency domain can be replaced by an infinite sequence of frequency response functions (FRFs) of different orders (Weiner and Spina, 1980). These FRFs are directly related to the DC component and different harmonics of the response (Weiner and Spina, 1980).

If the input is defined as a single harmonic periodic function with forcing amplitude $A$ and forcing frequency $\omega$

$x(t)=x_{s}+A \cos (\omega t)$

for infinite time, the output of a weakly nonlinear system is obtained as a sum of a DC component and the first, second, ... harmonics

$$
\begin{aligned}
& y(t)=y_{S}+y_{D C}+y_{I}+y_{I I}+\ldots \\
& \quad=y_{S}+y_{D C}+B_{I} \cos \left(\omega t+\varphi_{I}\right)+B_{I I} \cos \left(2 \omega t+\varphi_{I I}\right)+\ldots
\end{aligned}
$$

The DC component, which is responsible for the time-average performance of periodic processes and which is most essential in this paper, can be expressed as the following infinite series (Weiner and Spina, 1980):

$y_{\mathrm{DC}}=2\left(\frac{A}{2}\right)^{2} G_{2}(\omega,-\omega)+6\left(\frac{A}{2}\right)^{4} G_{4}(\omega, \omega,-\omega,-\omega)+\ldots$

where $G_{2}(\omega,-\omega)$ represents the asymmetrical second order frequency response function, $G_{4}(\omega, \omega,-\omega,-\omega)$ the asymmetrical fourth order FRF, etc.

For weakly nonlinear systems, the contributions of the higher other FRFs decrease with the increase of their order (Petkovska and Seidel-Morgenstern, 2013).

The dominant term of the DC component is proportional to the asymmetrical second order FRF, $G_{2}(\omega,-\omega)$ and the approximate value of the DC component can be easily calculated from (Marković et al., 2008)

$y_{D C} \approx 2\left(\frac{A}{2}\right)^{2} G_{2}(\omega,-\omega)$

In this way, the sign of the asymmetrical second order FRF $G_{2}(\omega,-\omega)$ defines the sign of the DC component. Consequently, in order to decide whether a particular periodic operation is favourable compared to the optimal steady-state operation, it is enough to derive and analyse the second order asymmetrical FRF (Marković et al., 2008). It is also possible to calculate approximately the magnitude of the improvement, by estimating the value of the second order asymmetrical FRF for chosen values of the forcing amplitude and forcing frequency. 
The procedure for derivation of the higher order FRFs is standard and can be found in Petkovska (2001), Petkovska et al. (2006), Petkovska and Do (1998), Petkovska and Marković (2006), Marković et al. (2008). The derivation process is recurrent, meaning that the first order FRFs have to be derived first, than the second order FRFs, etc. For our current application, we limit our derivations to the first order and the asymmetrical second order FRFs.

\subsection{NFR method for evaluation of process improvement in periodically operated chemical reactor}

Let us consider a continuously operated chemical reactor in which a simple reaction $A \rightarrow \operatorname{product}(s)$ takes place and one of the inputs is modulated periodically around a previously established steady-state. If conversion is of interest, the outlet concentration of the reactant $A$ can be considered as the output of interest. If the reactor is a nonlinear system, the mean value of the outlet concentration of the reactant $\left(c_{A}^{m}\right)$ during periodic operation will be different from the outlet steady-state concentration $\left(c_{A, S}\right)$. The difference $\Delta=c_{A}^{m}-c_{A, S}$, which is the indicator of the process improvement, depends on the type of nonlinearity. If $\Delta<0$, the periodic operation can be considered as favourable, as it corresponds to increased conversion, in comparison to the steady-state operation (Marković et al., 2008).

On the other hand, the difference between the time-average of the output of a periodically perturbed system and its steady-state value $(\Delta)$ is equal to the DC component of the outlet concentration. Since the dominant term of the DC component is proportional to the asymmetrical second order function $G_{2}(\omega,-\omega)$ (Petkovska et al., 2010), the time average response can be approximately estimated from this function

$\Delta=c_{A}^{m}-c_{A, S} \equiv c_{A, D C} \approx\left(2\left(\frac{A}{2}\right)^{2} G_{2}(\omega,-\omega)\right) c_{A, S}$

If the second order asymmetrical FRF is negative $\left(G_{2}(\omega,-\omega)<0\right)$ than the average concentration of the reactant $c_{A}^{m}$ will be lower than the steady-state outlet concentration $c_{A, S}(\Delta<0)$, i.e. the conversion will be increased.

By using Eq. (5) the magnitude of the process improvement can be approximately calculated, for any chosen forcing frequency and forcing amplitude.

\section{Mathematical model of a non-isothermal CSTR carrying out a simple reaction}

In this work, the NFR method is used to analyse the performance of a periodically operated non-isothermal CSTR in which a homogeneous $n$-th order reaction $A \rightarrow \operatorname{product}(s)$ takes place. The effects of modulating the concentration of the reactant in the inlet stream and the flow-rate of the feed stream on the reactor performance are analysed in this first part.

The rate law is

$r=k_{0} e^{-\left(E_{A} /(R T)\right)} c_{A}^{n}$

where $c_{A}$ is the reactant concentration, $T$ temperature, $k_{o}$ the preexponential factor in the Arrhenius equation, $E_{A}$ activation energy and $R$ the universal gas constant.

The material balance for the reactant $A$ is

$V \frac{d c_{A}}{d t}=F c_{A, i}-F c_{A}-k_{o} e^{-\left(E_{A} /(R T)\right)} c_{A}^{n} V$

and the energy balance

$V \rho c_{p} \frac{d T}{d t}=F \rho c_{p} T_{i}-F \rho c_{p} T+\left(-\Delta H_{R}\right) k_{o} e^{-\left(E_{A} /(R T)\right)} c_{A}^{n} V-U A_{w}\left(T-T_{J}\right)$ where $t$ is time, $F$ the volumetric flow-rate of the reaction stream, $V$ the volume of the reactor, $\Delta H_{R}$ heat of reaction, $U$ the overall heat transfer coefficient, $A_{w}$ the surface area for heat exchange, $\rho$ density, $c_{p}$ specific heat capacity. The following subscripts are used in the balance equations: $i$ for inlet and $J$ for heating/cooling fluid in the reactor jacket.

It is assumed that volume is constant ( $V=$ const), i.e. that the inlet and outlet flow-rates are equal, that the temperature of the heating/cooling fluid does not change from inlet to outlet and that all physical and chemical properties are independent on temperature ( $\rho c_{p}=$ const, $\Delta H_{R}=$ const).

In steady-state, the material and energy balances are given with the following expressions:

$$
\begin{aligned}
& \frac{c_{A i, S}}{c_{A, S}}=1+k_{o} e^{-\left(E_{A} /\left(R T_{S}\right)\right)} c_{A, S}^{n-1} \frac{V}{F_{S}}=1+\alpha \\
& \frac{T_{i, S}}{T_{S}}=1-\frac{\left(-\Delta H_{R}\right) k_{o} e^{-\left(E_{A} /\left(R T_{S}\right)\right)} c_{A, S}^{n}}{\rho c_{p} T_{S}} \frac{V}{F_{S}}+\frac{U A_{w}}{F_{S} \rho c_{p}}-\frac{U A_{w} T_{J, S}}{F_{S} \rho c_{p} T_{S}}=1+\beta+S t-\delta
\end{aligned}
$$

where subscript $s$ denotes the steady-state values and the following auxiliary parameters have been introduced:

$\alpha=k_{o} e^{-\left(E_{A} /\left(R T_{S}\right)\right)} c_{A, S}^{n-1} \frac{V}{F_{S}}, \beta=\frac{\Delta H_{R} k_{o} e^{-\left(E_{A} /\left(R T_{S}\right)\right)} c_{A, S}^{n}}{\rho c_{p} T_{S}} \frac{V}{F_{S}}, \delta=\frac{U A_{W} T_{J, S}}{F_{S} \rho c_{p} T_{S}}$,

$\gamma=\frac{E_{A}}{R T_{s}}, S t=\frac{U A_{w}}{F_{s} \rho c_{p}}$

These parameters are functions of the physical parameters of the reactor, as well as the steady-state values of the concentration and temperature in the reactor $\left(\mathrm{c}_{\mathrm{A}, s}\right.$ and $\left.T_{s}\right)$.

For analysis in frequency domain, it is convenient to transform the model equations into dimensionless form, by introducing dimensionless variables as relative deviations from their steadystate values. The definitions of the dimensionless variables are given in Table 1.

Hereby $T_{i, s}$ and $T_{J, s}$ represent constant steady-state values of the temperatures of the feed stream and in the jacket. Since these temperature are not modulated, the corresponding $\theta_{i}$ and $\theta_{J}$ are zero in this study.

After introducing the dimensionless variables and the auxiliary parameters in model Eqs. (7) and (8), the model equations become:

$$
\begin{aligned}
\frac{d C}{d \tau}= & (1+\alpha)(\Phi+1)\left(C_{i}+1\right)-(\Phi+1)(C+1) \\
& -k_{o} C_{A, S}^{n-1} \frac{V}{F_{S}} e^{-\left(E_{A} /\left(R T_{S}(\theta+1)\right)\right)}(1+C)^{n}
\end{aligned}
$$

$$
\begin{gathered}
\frac{d \theta}{d \tau}=(1+\beta+S t-\delta)(\Phi+1)\left(\theta_{i}+1\right)-(\Phi+1)(\theta+1)-S t(\theta+1)+\delta\left(\theta_{J}+1\right) \\
-\frac{\Delta H_{R} k_{o} C_{A, s}^{n} V}{\rho C_{p} T_{S} F_{S}} e^{-\left(E_{A} /\left(R T_{S}(\theta+1)\right)\right)}(1+C)^{n}
\end{gathered}
$$

Table 1

Definitions of the dimensionless variables.

\begin{tabular}{ll}
\hline Inlet concentration of the reactant & $C_{i}=\frac{c_{A i}-c_{A i, s}}{C_{A i, s}}$ \\
Flow-rate & $\Phi=\frac{F-F_{s}}{F_{s}}$ \\
Outlet concentration of the reactant & $C=\frac{c_{A}-c_{A, s}}{C_{A, s}}$ \\
Inlet temperature & $\theta_{i}=\frac{T_{i}-T_{i, s}}{T_{i, s}}$ \\
Temperature in the reactor & $\theta=\frac{T-T_{s}}{T_{s}}$ \\
Temperature of the heating/cooling fluid & $\theta_{J}=\frac{T_{J}-T_{J, s}}{T_{J, s}}$ \\
Time & $\tau=\frac{t}{V / F_{s}}$ \\
Frequency & $\omega=\omega_{d} \frac{V}{F_{s}}$ \\
\hline
\end{tabular}


For applying the NFR method, all nonlinearities in the model equations should be given in the polynomial form (Weiner and Spina, 1980). After expanding the nonlinear terms $e^{-\left(E_{A} /\left(R T_{S}(\theta+1)\right)\right)}$ and $(1+C)^{n}$ in the Taylor series form, the dimensionless model equations are transformed into the following form:

$$
\begin{aligned}
\frac{d C}{d \tau}= & (1+\alpha)(\Phi+1)\left(C_{i}+1\right)-(\Phi+1)(C+1) \\
& -\alpha\left(1+n C+\gamma \theta+n \gamma C \theta+\left(\frac{\gamma^{2}}{2}-\gamma\right) \theta^{2}+\frac{1}{2} n(n-1) C^{2}+\ldots\right)
\end{aligned}
$$

$$
\begin{aligned}
\frac{d \theta}{d \tau}= & (1+\beta+S t-\delta)(\Phi+1)\left(\theta_{i}+1\right)-(\Phi+1)(\theta+1)-S t(\theta+1)+\delta\left(\theta_{J}+1\right) \\
& -\beta\left(1+n C+\gamma \theta+n \gamma C \theta+\left(\frac{\gamma^{2}}{2}-\gamma\right) \theta^{2}+\frac{1}{2} n(n-1) C^{2}+\ldots\right)
\end{aligned}
$$

The Taylor series expansions of the nonlinear terms $e^{-\left(E_{A} /\left(R T_{s}(\theta+1)\right)\right)}$ and $(1+C)^{n}$ in the dimensionless model equations are given in Appendix A. In Eqs. (14) and (15) only the first and second order terms are shown.

\section{Frequency response functions of the non-isothermal CSTR}

\subsection{Definitions of the FRFs}

In the case of a single input modulation, the non-isothermal CSTR represents a nonlinear system with one modulated input and two outputs. The modulation of a chosen input, for the nonisothermal reactor, will cause changes of both the concentration and the temperature in the reactor. Consequently, in order to describe the behaviour of non-isothermal CSTR for a single input modulation, for each modulated input it is necessary to derive two sets of FRFs, as presented in Fig. 1.

The following notations are used in Fig. $1 X$ for dimensionless input modulation (in this work, inlet concentration or flow-rate), $C$ dimensionless outlet concentration of reactant, $\theta$ dimensionless outlet temperature, $G_{n X}{ }^{n}$ the $n$-th order FRF correlating the outlet concentration to the modulated input $X$ and $F_{n, X}$ the $n$-th order FRFs correlating the outlet temperature to the modulated input $X$. Accordingly, the following four sets of FRFs need to be derived

Set 1: FRFs which correlate the outlet concentration of the reactant with the modulated inlet concentration $\left(G_{1, C}(\omega), G_{2, C C}\right.$ $(\omega,-\omega), \ldots)$.

Set 2: FRFs which correlate the outlet temperature with the modulated inlet concentration $\left(F_{1, C}(\omega), F_{2, C C}(\omega,-\omega), \ldots\right)$.

Set 3: FRFs which correlate the outlet concentration with the modulated flow-rate $\left(G_{1, F}(\omega), G_{2, F}(\omega,-\omega), \ldots\right)$.

Set 4: FPFs which correlate the outlet temperature with the modulated flow-rate $\left(F_{1, F}(\omega), F_{2, F F}(\omega,-\omega), \ldots\right)$.

From the aspect of process improvement, the change of the outlet temperature is not of particular interest, but it might be relevant from the aspect of safety and equipment limitations. In that case, the change of outlet temperature owing to periodic operation could be estimated in an analogous way as the outlet concentration, from the asymmetrical second order FRF which

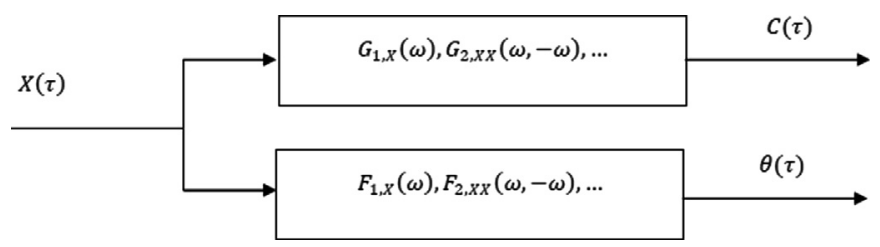

Fig. 1. Block diagram of a non-isothermal CSTR for single input modulation. correlates the outlet temperature with the modulated input.

$\Delta_{T}=\left(2\left(\frac{A}{2}\right)^{2} F_{2, X X}(\omega,-\omega)\right) T_{S}$

The $F$-functions are not subject of the analysis reported here, since we are mainly interested in the outlet concentration and the conversion change of the periodically operated non-isothermal CSTR. However, the F-functions have to be derived since they are necessary in the derivation process of the $G$-functions. Therefore, the derivation of the $F$-functions will be provided without further analysis.

\subsection{Derivation of the FRFs}

The basic steps of the procedure for derivation of the frequency response functions are

1. The input modulation (inlet concentration $C_{i}(\tau)$ or flow-rate $\Phi(\tau))$ is defined in the form of a co-sinusoidal function.

2. The outlet concentration $C(\tau)$ and temperature $\theta(\tau)$ are expressed in the Volterra series form.

3. The expression for the modulated input $\left(C_{i}(\tau)\right.$ or $\left.\Phi(\tau)\right)$ and for $C(\theta), \tau(\theta)$ from step 1 and step 2, are substituted into the corresponding dimensionless model equations (Eqs. (14) and (15)).

4. The method of harmonic probing is applied to the equations obtained in step 3, i.e., the terms with the same amplitude and frequency are collected and equated to zero.

5. The equations obtained in step 4 are solved.

The final expressions for the asymmetrical second order FRFs which correlate the outlet concentration with the modulated input are given below while and the most important steps of the derivation procedure are given in Appendix B.

4.2.1. Periodic operation with modulation of the inlet concentration

Starting from the dimensionless model Eqs. (14) and (15), in which all input variables except the inlet concentration are set to zero $\left(\Phi=0, \theta_{i}=0, \theta_{J}=0\right)$, the $G_{C^{-}}$and $F_{C^{-}}$functions are derived. Both first order and asymmetrical second order FRFs are given in Appendix B.1, together with the main steps of the derivation procedure.

Here we consider only the asymmetrical second order FRF corresponding to the outlet concentration, $G_{2, c c}(\omega,-\omega)$, as it contains information regarding the conversion change owing to periodic operation. The final expression of $G_{2, c c}(\omega,-\omega)$ is

$$
\begin{aligned}
& G_{2, C c}(\omega,-\omega)=-\frac{1}{2} \frac{\alpha(1+S t)}{1+n \alpha+\beta \gamma+n \alpha S t+S t} \\
& \quad \times \frac{(1+\alpha)^{2} \Lambda}{\left[(1+n \alpha+\beta \gamma+n \alpha S t+S t)-\omega^{2}\right]^{2}+\omega^{2}(2+\beta \gamma+S t+n \alpha)^{2}}
\end{aligned}
$$

where the term in the numerator $\Lambda$ is defined as:

$\Lambda=n(n-1) \omega^{2}+\left[(1+S t)^{2}-2 \beta^{2} \gamma\right] n^{2}-\left[(1+S t+\beta \gamma)^{2}\right] n=\Lambda_{1} \omega^{2}+\Lambda_{2}$

\subsubsection{Periodic operation with modulation of the flow-rate}

The $G_{F}$ and $F_{F}$-functions are again derived starting from the dimensionless model Eqs. (13) and (14), in which, this time, all input variables except the flow-rate are set to zero $\left(C_{i}=0, \theta_{i}=0, \theta_{J}=0\right)$. The first order and asymmetrical second order FRFs are given in Appendix B.2, together with the main steps of the derivation procedure.

Here we give the final expression for the asymmetrical second order FRF which correlates the outlet concentration to the 
modulated flow-rate

$$
\begin{aligned}
& G_{2, F F}(\omega,-\omega)=-\frac{1}{2} \frac{1}{1+n \alpha+\beta \gamma+n \alpha S t+S t} \\
& \quad \times \frac{\Omega}{\left[(1+n \alpha+\beta \gamma+n \alpha S t+S t)-\omega^{2}\right]^{2}+\omega^{2}(2+\beta \gamma+S t+n \alpha)^{2}}
\end{aligned}
$$

where the term $\Omega$ in the numerator can be expressed in the form of a polynomial with respect to the forcing frequency

$\Omega=\Omega_{1} \omega^{2}+\Omega_{2}$

with $\Omega_{1}$ and $\Omega_{2}$ which are complex functions of the reaction order $n$ and the model parameters $\alpha, \beta, \gamma, \delta$ and $S t$

$$
\begin{aligned}
\Omega_{1}= & \alpha^{3}(1+S t) n^{2}+\alpha^{2}(1+S t)(2-\alpha+2 \gamma(\beta+S t-\delta)) n+2 \alpha(1+S t+\beta \gamma) \\
& +\alpha \gamma(\gamma-2)(1+S t)(\beta+S t-\delta)^{2} \\
\Omega_{2}= & {\left[\alpha^{3} \gamma(\gamma-2)(1+S t)(S t-\delta)^{2}-2 \alpha^{3} \gamma(S t-\delta)(1+S t)\right.} \\
& +\alpha^{3}(1+S t)(1+S t-\gamma S t+\gamma \delta)^{2} \\
& \left.+2 \alpha^{3} \gamma(1+S t)(1+S t-\gamma S t+\gamma \delta)(S t-\delta) n^{2}\right] \\
& +\left[2 \alpha^{2}(1+S t+\beta \gamma)(1+S t-\gamma S t+\gamma \delta)(1+S t)\right. \\
& -2 \alpha^{2} \gamma(1+S t)(\beta+S t-\delta)-2 \alpha^{2} \gamma(S t-\delta)(1+\beta \gamma+S t) \\
& +2 \alpha^{2} \gamma(\gamma-2)(1+S t)(S t-\delta)(\beta+S t-\delta) \\
& -\alpha^{3}(1+S t)(1+S t-\gamma S t+\gamma \delta)^{2} \\
& \left.+2 \alpha^{2} \gamma(1+S t)(1+S t-\gamma S t+\gamma \delta)(\beta+S t-\delta)\right] n \\
& +\left[2 \alpha(1+S t+\beta \gamma)^{2}(1+S t-\gamma S t+\gamma \delta)-2 \alpha \gamma(1+\beta \gamma+S t)(\beta+S t-\delta)\right. \\
& \left.+\alpha\left(\gamma^{2}-2 \gamma\right)(1+S t)(\beta+S t-\delta)^{2}\right]
\end{aligned}
$$

\subsection{Stability analysis}

Considering that the NFR method is applicable only for stable systems and the fact that non-isothermal CSTR can be unstable, it is important to determine the domain in which the system is stable.

By analysis of the characteristic equation which corresponds to the linear model, we can determine the domain of stability and oscillatority. The characteristic equation of the linearized model can be obtained by equating the denominator of the first order FRF $\left(G_{1 C}, F_{1 C}, G_{1 F}\right.$ and $F_{1 C}$, given in Appendix B all have the same denominator) with zero, after replacing $j \omega$ with the Laplace complex variable $s$. For the non-isothermal CSTR defined by model Eqs. (11) and (12) the characteristic equation of the system is the following second-order equation:

$s^{2}+s(2+\beta \gamma+S t+n \alpha)+(1+n \alpha+\beta \gamma+n \alpha S t+S t)=0$

The roots of this characteristic equation are

$p_{1,2}=A_{p s} \pm \sqrt{\left(A_{p s}^{2}-B_{p s}\right)}$

where

$A_{p s}=-\frac{(2+n \alpha+S t+\beta \gamma)}{2}$ and $B_{p s}=(1+n \alpha+\beta \gamma+n \alpha S t+S t)$

The necessary and sufficient condition that a linear system is stable is that all roots of characteristic equation are negative or have negative real parts (Douglas, 1972). Although this stability condition is valid only for linear systems, this analysis can provide valuable information about the stability of a nonlinear system in the vicinity of the steady-state, based on the Lyapunov theorem (Douglas, 1972). The oscillatority of the system is also determined by the position of the roots of the characteristic equation. If all roots of the characteristic equations are real, the system will be non-oscillatory, otherwise, if the roots of characteristic equations are conjugate-complex, the system will be oscillatory.

Based on all this, we can conclude that for

$A_{p s}<0$ and $B_{p s}>0$ and $A_{p s}^{2}>B_{p s}$ - the system is stable and nonoscillatory,

$A_{p s}<0$ and $B_{p s}>0$ and $A_{p s}^{2}<B_{p s}$ - the system is stable and oscillatory,

$A_{p s}>0$ and $B_{p s}>0$ and $A_{p s}^{2}>B_{p s}$ - the system is unstable and nonoscillatory,

$A_{p s}>0$ and $B_{p s}>0$ and $A_{p s}^{2}<B_{p s}$ - the system is unstable and oscillatory.

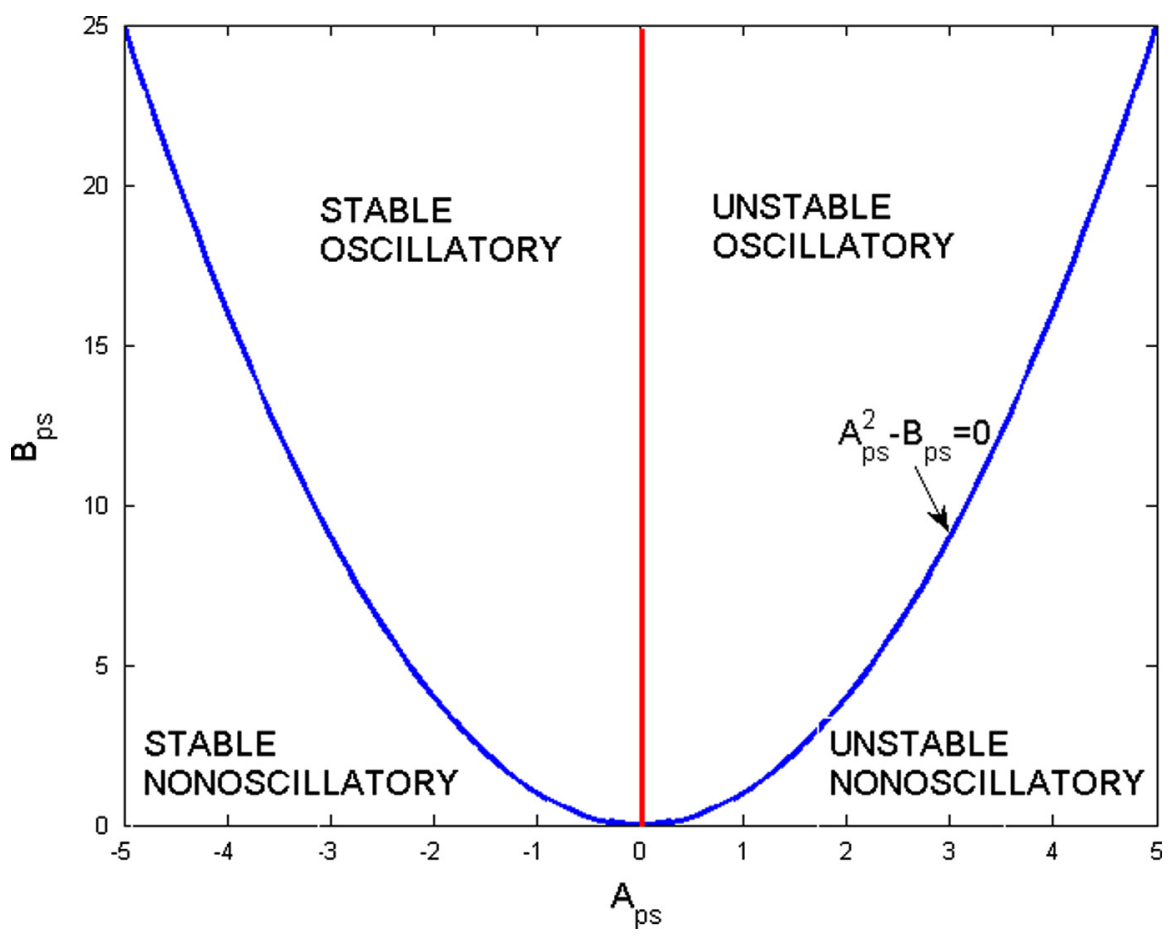

Fig. 2. Areas of stability and oscillatority for a non-isothermal CSTR. 
The areas of stability and oscillatority, depending on the auxiliary stability parameters $A_{p s}$ and $B_{p s}$ are graphically presented in Fig. 2.

The nonlinear frequency response method and the concept of higher order FRFs can be applied only in the domain in which the system is stable, i.e. for $A_{p s}<0, B_{p s}>0$.

If the characteristic equation of the non-isothermal CSTR (Eq. (23)) is given in its standard form (Douglas, 1972)

$s^{2}+2 \xi \omega_{n} s+\omega_{n}^{2}=0$

the commonly used model parameters, damping coefficient $\xi$ and natural frequency $\omega_{n}$, can be defined. It is a well known fact that a stable oscillatory system, with a damping coefficient $\xi$ less than 0.707 exhibits resonance (amplification of the inlet modulation for some input frequencies) (Douglas, 1972). The frequency at which the amplitude of the outlet is maximal is called resonant frequency and depends on $\xi$ and the natural frequency $\omega_{n}$ of the oscillatory system (Douglas, 1972)

$\omega_{r}=\omega_{n} \sqrt{1-2 \xi^{2}}$

It can be shown that this resonant frequency can be calculated from the stability parameters $A_{p s}$ and $B_{p s}$, in the following way

$\omega_{r}=\sqrt{B_{p s}-2 A_{p s}^{2}}$

Previous investigations suggest that the maximum improvement of a forced periodic operation in comparison with the optimal steady-state operation can be achieved around the resonant frequency (Ritter and Douglas, 1970).

\section{Analysis of the signs of the asymmetrical second order FRFs}

\subsection{Modulation of the inlet concentration}

By using the stability parameters $A_{p s}$ and $B_{p s}$ defined in Eq. (25), Eq. (17) for the second order asymmetrical FRF $G_{2, C C}$ $(\omega,-\omega)$ can be written in the following way:

$G_{2, C C}(\omega,-\omega)=-\frac{1}{2} \frac{\alpha(1+S t)}{B_{p s}} \frac{(1+\alpha)^{2} \Lambda}{4 A_{p s}^{2} \omega^{2}+\left(B_{p s}-\omega^{2}\right)^{2}}$

It is necessary to point out that all auxiliary parameters $(\alpha, \gamma, S t, \delta)$ are always positive, except $\beta$ which is positive for endothermic and negative for exothermic reactions.

Considering that for a stable system $A_{p s}<0, B_{p s}>0$, it can easily be concluded that sign of the second order asymmetrical FRF $G_{2, C C}(\omega,-\omega)$ depends only on the sign of the term $\Lambda$ and that:

$\operatorname{sign}\left(G_{2, C C}(\omega,-\omega)\right)=-\operatorname{sign}(\Lambda)$

Based on this, the sign analysis of FRF $G_{2, C C}(\omega,-\omega)$ is reduced to sign analysis of the term $\Lambda$, which depends on the reaction order $n$, values of the auxiliary parameters $\beta, \gamma$, St and the forcing frequency $\omega$. The reaction order $n$ and the values of the auxiliary parameters are characteristics of the particular system. Considering this and the fact that forcing frequency can be chosen, it is interesting to analyse how the sign of the second order asymmetrical FRF depends on the forcing frequency $\omega$.

The sign of $G_{2, C C}(\omega,-\omega)$ will change at certain frequency, if the following equation has real solutions:

$$
\begin{aligned}
& G_{2, C C}(\omega,-\omega)=0 \Leftrightarrow \Lambda=0 \Leftrightarrow \\
& \quad n(n-1) \omega^{2}+\left[(1+S t)^{2}-2 \beta^{2} \gamma\right] n^{2}-\left[(1+S t+\beta \gamma)^{2}\right] n=0
\end{aligned}
$$

If Eq. (31) has no real solution for $\omega$, the function $G_{2, C C}(\omega,-\omega)$ is either positive or negative in the whole frequency range.
The solutions of Eq. (31) are

$$
\omega_{1,2}= \pm \omega_{C}= \pm \sqrt{\frac{(1+S t+\beta \gamma)^{2}-n\left((1+S t)^{2}-2 \beta^{2} \gamma\right)}{n-1}}
$$

These solutions will be real if the numerator and denumenator of the rational function under the square root have the same sign. The sign of the denominator depends on the reaction order and changes for $n=1$. The sign of the numerator also depends on the reaction order and changes for

$n=n_{C}=\frac{(1+S t+\beta \gamma)^{2}}{(1+S t)^{2}-2 \beta^{2} \gamma}$

The results of the sign analysis in respect to the reaction order and the frequency range are summarized in the Table 2.

\subsection{Modulation of the flow-rate}

The final expression for the asymmetrical second order FRF $G_{2, F F}(\omega,-\omega)$, defined by Eq. (19) can again be transformed into more appropriate form for sign analysis, by using the definitions of the stability parameters $A_{p s}$ and $B_{p s}$ :

$G_{2, F F}(\omega,-\omega)=-\frac{1}{2 B_{p s}} \frac{\Omega}{4 A_{p s}^{2} \omega^{2}+\left(B_{p s}-\omega^{2}\right)^{2}}$

In principle, the sign of $G_{2, F F}(\omega,-\omega)$, depends on the reaction order $n$, forcing frequency $\omega$, and the auxiliary parameters $\alpha, \beta, \gamma, \delta$ and St. From Eq. (34) it can be easily concluded that the sign of $G_{2, F F}(\omega,-\omega)$ depends only on the sign of the auxiliary function in the numerator, $\Omega$ in the following way

$\operatorname{sign}\left(G_{2, F F}(\omega,-\omega)\right)=-\operatorname{sign}(\Omega)$

Thus, the sign analysis of $G_{2, F F}(\omega,-\omega)$ can be further reduced to sign analysis of $\Omega$, which was defined in Eq. (20) as $\Omega=\Omega_{1} \omega^{2}+\Omega_{2}$ ( $\Omega_{1}$ and $\Omega_{2}$ are complex functions of the reaction order, defined in Eqs. (21) and (22)).

The frequency for which the function $G_{2, F F}(\omega,-\omega)$ changes its sign is obtained from the following condition:

$G_{2, F F}(\omega,-\omega)=0 \Leftrightarrow \Omega=0 \Leftrightarrow \Omega_{1} \omega^{2}+\Omega_{2}=0$

If the solutions of Eq. (36)

$\omega_{1,2}= \pm \omega_{F}= \pm \sqrt{-\frac{\Omega_{2}}{\Omega_{1}}}$

are real, which will happen if $\Omega_{1}$ and $\Omega_{2}$ have opposite signs, $G_{2, F F}(\omega,-\omega)$ will change its sign for $\omega_{F}$, while if they are complexconjugates, $G_{2, F F}(\omega,-\omega)$ will be positive or negative in the whole frequency range. A summary of the sign analysis for the $G_{2, F F}(\omega,-\omega)$ function is given in Table 3.

Table 2

A summary of the sign analysis for the $G_{2, C C}(\omega,-\omega)$ function for forced periodic operation of a non-isothermal CSTR (attractive are negative signs).

\begin{tabular}{lll}
\hline Condition & Frequency range & Sign of $G_{2, C C}(\omega,-\omega)$ \\
\hline$n=0$ & $\forall \omega$ & 0 \\
$n<n_{C}$ and $n<0$ & $\forall \omega$ & negative \\
$n<n_{C}$ and $0<n<1$ & $\forall \omega$ & positive \\
$n_{C}<1$ and $n=1$ & $\forall \omega$ & negative \\
$n<n_{C}$ and $n>1$ & $\omega<\omega_{C}$ & positive \\
& $\omega>\omega_{C}$ & negative \\
$n>n_{C}$ and $n<0$ & $\omega<\omega_{C}$ & positive \\
$n>n_{C}$ and $0<n<1$ & $\omega>\omega_{C}$ & negative \\
$\frac{1}{n_{C}}<1$ and $n=1$ & $\omega<\omega_{C}$ & negative \\
$n>n_{C}$ and $n>1$ & $\omega>\omega_{C}$ & positive \\
\hline
\end{tabular}


Table 3

A summary of the sign analysis for the $G_{2, F F}(\omega,-\omega)$ function for forced periodic operation of a non-isothermal CSTR (attractive are negative signs).

\begin{tabular}{llll}
\hline Sign of $\Omega_{1}$ & Sign of $\Omega_{2}$ & Frequency range & Sign of $G_{2, F F}(\omega,-\omega)$ \\
\hline positive & negative & $\begin{array}{l}\omega<\omega_{F} \\
\omega>\omega_{F}\end{array}$ & positive \\
& & negative \\
negative & positive & $\omega<\omega_{F}$ & negative \\
& & $\forall \omega \omega_{F}$ & positive \\
positive & positive & $\forall \omega$ & negative \\
negative & negative & $\forall \omega$ & positive \\
zero & negative & $\forall \omega$ & positive \\
& positive & $\forall \omega$ & negative \\
positive & zero & $\forall \omega$ & negative \\
negative & & & positive \\
\hline
\end{tabular}

Table 4

Parameters for the numerical example.

\begin{tabular}{ll}
\hline Parameter & Value \\
\hline Reaction order, $n$ & 1 \\
Volume of the reactor, $V\left[\mathrm{~m}^{3}\right]$ & 1 \\
Preexponential factor of the reaction rate constant, $k_{o}[1 / \mathrm{min}]$ & $1 \times 10^{10}$ \\
Activation energy, $E_{A}[\mathrm{~kJ} / \mathrm{kmol}]$ & 69256 \\
Heat of reaction, $\Delta H_{R}[\mathrm{~kJ} / \mathrm{kmol}]$ & -543920 \\
Heat capacity, $\rho c_{p}\left[\mathrm{~kJ} / \mathrm{K} / \mathrm{m}^{3}\right]$ & $4.184 \times 10^{3}$ \\
Steady-state flow-rate, $F_{S}\left[\mathrm{~m}^{3} / \mathrm{min}\right]$ & 1 \\
Steady-state inlet concentration, $c_{A i, s}\left[\mathrm{kmol} / \mathrm{m}^{3}\right]$ & 2 \\
Steady-state inlet temperature, $T_{i, s}[\mathrm{~K}]$ & 323 \\
Steady-state temperature of the coolant, $T_{J, s}[\mathrm{~K}]$ & 365 \\
\hline
\end{tabular}

\section{Numerical example}

\subsection{Definition of the numerical example}

In order to clarify the results given in Sections 4 and 5, a numerical example is chosen for simulation of the asymmetrical second order FRFs and analysis of their sign, and for comparison of the results obtained by the NFR method and with numerical simulation. The values of the model parameters used for simulations are given in Table 4. The parameters listed in Table 4 correspond to an exothermal first-order reaction and were taken from a classical textbook by Marlin, (2000) (Example 3.10).

For the numerical example and the steady state input variables defined in Table 4, it was determined that there exists only one steady state solution, defined by $c_{A, S}=0.3466 \mathrm{kmol} / \mathrm{m}^{3}$ and $T_{s}=388 \mathrm{~K}$. It should be pointed out that this steady-state has not been optimized.

\subsection{Stability analysis for the numerical example}

Using the parameter values defined in Table 4, the following values of the stability parameters are calculated using Eq. (24): $A_{p s}=-0.7<0, \quad B_{p s}=32>0, A_{p s}^{2}-B_{p s}=31<0$. Based on these values we can conclude that the system is oscillatory stable, therefore application of the NFR method makes sense for this forced periodically operated non-isothermal CSTR.

The damping coefficient for this system, which was calculated based on Eqs. (23) and (26), is quite low, $\xi=0.126$, so extensive resonance is expected. The resonant frequency is $\omega_{r}=5.53$ (Eq. (28)).

It is also important to choose the amplitudes of the input modulation for which the system remains stable. In Figs. 3 and 4 we show the stability parameter $A_{p s}$ and $B_{p s}$ vs. $c_{A i, s}$ and $F_{S}$, respectively, with steady-state values of all other inputs fixed at their values given in Table 4. As it can be seen from these figures, the parameter $A_{p s}$ is negative and the parameter $B_{p s}$ positive for all values of $c_{A i, s}$ and $F_{S}$, i.e. the amplitudes of the inlet concentration and flow-rate are not limited from the stability aspect. However, considering physical restrictions, the maximal forcing amplitudes are $100 \%$ (the inputs cannot be negative).

\subsection{Simulation of the asymmetrical second order FRFS}

In Figs. 5 and 6, graphical representations of the asymmetrical second order FRFs $G_{2, C C}(\omega,-\omega)$ and $G_{2, F F}(\omega,-\omega)$ vs. frequency are given.

From the graphical representation of the asymmetrical second order FRFs (Figs. 5 and 6) for this investigated numerical example and from Eqs. (17)-(22) or (29) and (34), it can be concluded that

- The asymmetrical second order FRFs tend to zero for high frequencies which is in accordance with conclusions of our previous investigations that high frequency modulations have no influence on the reactor performance.

- For low forcing frequencies the asymmetrical second order FRFs tend to asymptotic values

$$
\begin{aligned}
& \lim _{\omega \rightarrow 0} G_{2, C C}(\omega,-\omega)=-\frac{1}{2} \frac{\alpha(1+\alpha)^{2}(1+S t)}{B_{p s}^{3}} \Lambda_{2} \\
& \lim _{\omega \rightarrow 0} G_{2, F F}(\omega,-\omega)=-\frac{1}{2 B_{p s}^{3}} \Omega_{2}
\end{aligned}
$$

- $G_{2, c c}(\omega,-\omega)$ is negative in the whole frequency range, so an increase of conversion is expected with periodic modulation of the inlet concentration in the whole frequency range. This is an expected results, as for our numerical example the parameter $n_{C}=0.4348$, so $n=1>n_{C}$. From the results of sign analysis given in Table 2, for this case negative values of the $G_{2, c c}(\omega,-\omega)$ function are expected in the whole frequency range.

- $G_{2, F F}(\omega,-\omega)$ is negative for dimensionless forcing frequencies $\omega<6.71$ and positive for $\omega>6.71$ Consequently, increase of conversion can be expected only with flow modulations with frequencies lower than 6.71. These results are also in accordance with the results obtained by sign analysis in Section 5.2. From the values of parameters $\Omega_{1}=-510<0$ and $\Omega_{2}=2.3 \times 10^{4}>0$ and Table 3 , it can also be concluded that the $G_{2, F F}(\omega,-\omega)$ function changes its sign for $\omega=\omega_{F}=6.71$, and that for $\omega=\omega_{F}=6.71$, the FRF $G_{2, F F}(\omega,-\omega)$ is negative, and for $\omega>\omega_{F}=6.71$ it is positive.

- The highest improvement in both cases of periodical modulation is expected when the forcing frequency has a value close to the resonant frequency $\left(\omega_{r}=5.53\right)$ for which the asymmetrical second order FRFs have extensive minima.

- From the graphical representation of the FRFs, we can expect that larger improvements can be achieved with modulation of the inlet concentration than with modulation of the flow-rate for the same values of frequency and forcing amplitude.

\subsection{Comparison with results obtained by numerical integration}

The DC components of the outlet concentration, calculated approximately by application of the NFR method, are compared with the results obtained by numerical integration of the model Eqs. (7) and (8), with the model parameters defined in Table 4 and for modulation of the inlet concentration of the reactant and flowrate. The equations were solved by using a standard Matlab function ode15s. 
As illustration, in Fig. 7 we show some simulation results (the outlet concentration and outlet temperature vs. time). This figure corresponds to modulation of the inlet concentration, with amplitude $A=50 \%$ and forcing frequency equal to the resonant frequency $\left(\omega_{d}=5.53 \mathrm{rad} / \mathrm{min}\right)$. The starting steady-state values and the mean values during periodic operation, are also shown.

For the case presented in Fig. 7 the outlet concentration of the reactant oscillates between 0.03 and $0.66 \mathrm{kmol} / \mathrm{m}^{3}$, while the temperature oscillates between 369 and $425 \mathrm{~K}$. The mean value of the outlet concentration $c_{A}^{m}=0.3083 \mathrm{kmol} / \mathrm{m}^{3}$ is lower than the steady state value $\left(0.3466 \mathrm{kmol} / \mathrm{m}^{3}\right)$, which makes the

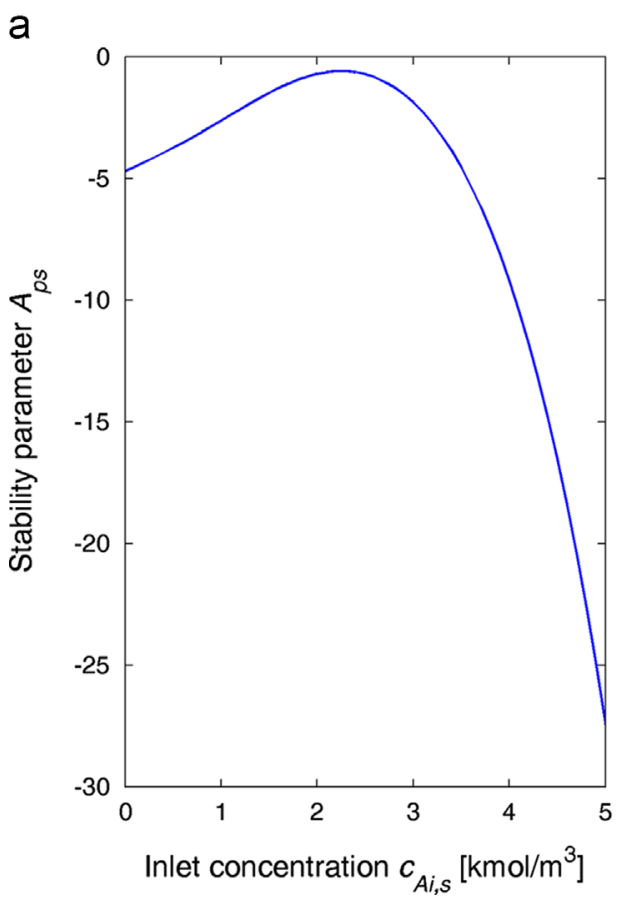

periodic operation attractive. It is interesting to notice that, although the temperature response is also highly nonlinear, the mean temperature $T^{m}=388.8 \mathrm{~K}$ is close to the steady state value $T_{s}=388 \mathrm{~K}$.

An overview comparing the results of the NFR method with the ones obtained by numerical simulations is given in Table 5. The difference between the mean outlet concentration of the reactant, when the selected input is periodically modulated, and the outlet concentration of the reactant for steady-state operation

$\Delta_{\text {num }}=c_{A}^{m}-c_{A, s}$

b

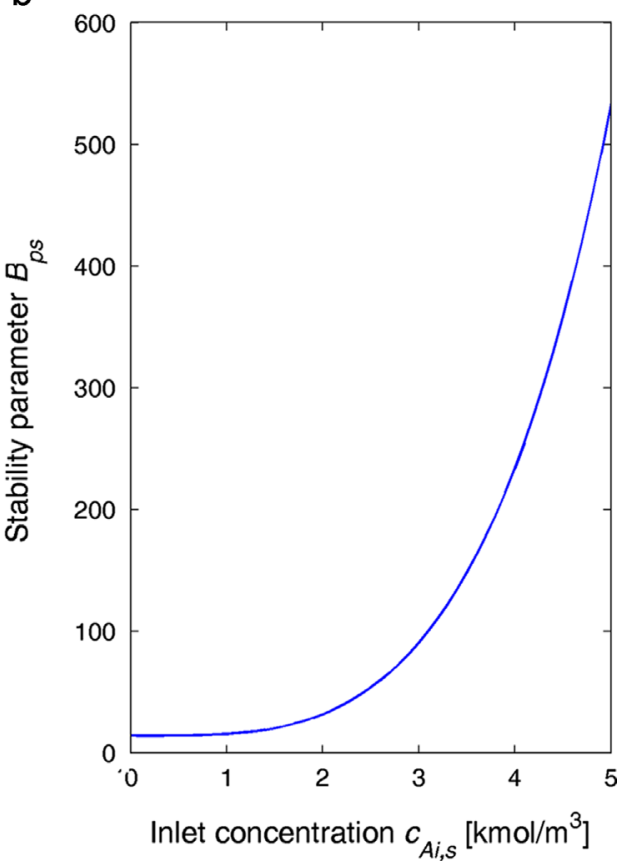

Fig. 3. Stability parameters $A_{p s}$ (a) and $B_{p s}$ (b) vs. steady-state inlet concentration.

a

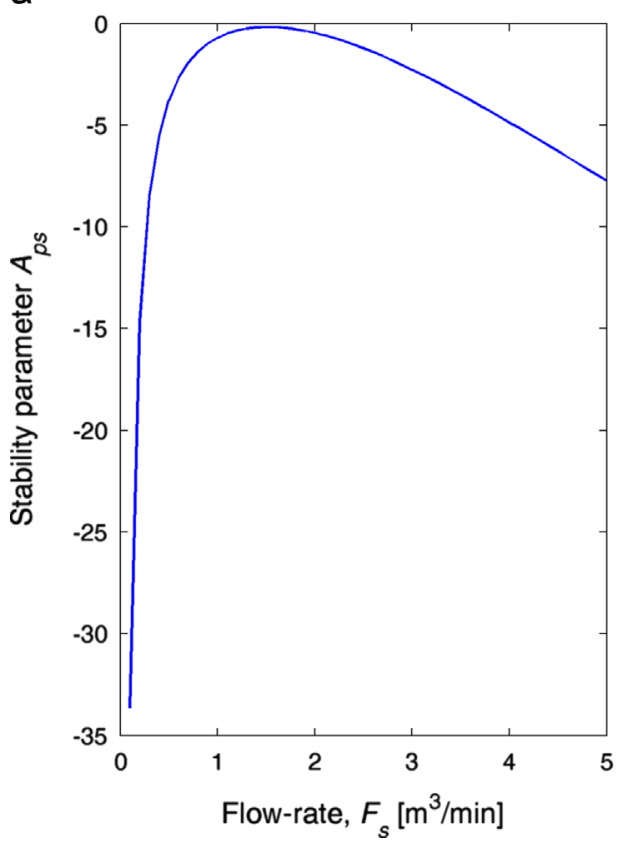

b

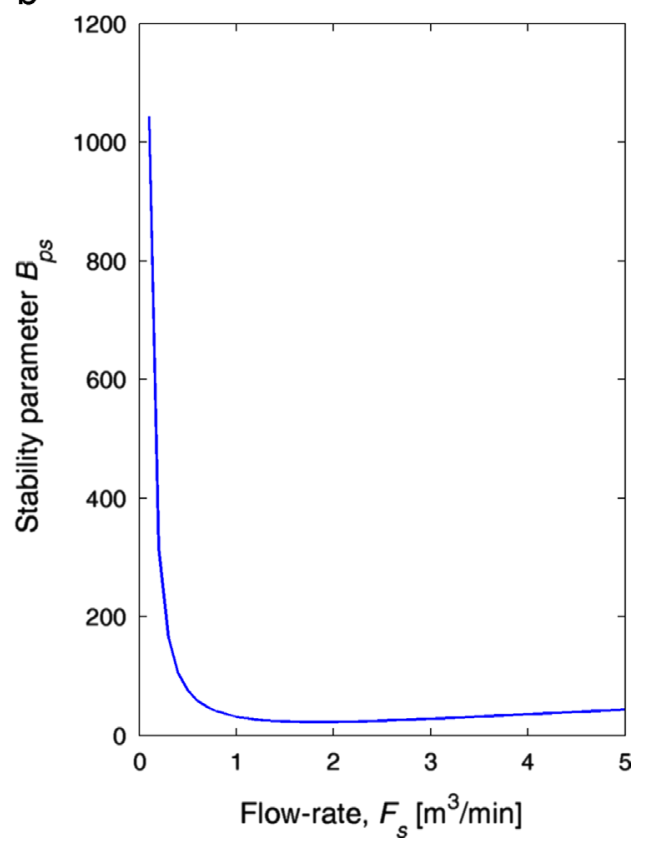

Fig. 4. Stability parameters $A_{p s}$ (a) and $B_{p s}$ (b) vs. steady-state flow-rate. 


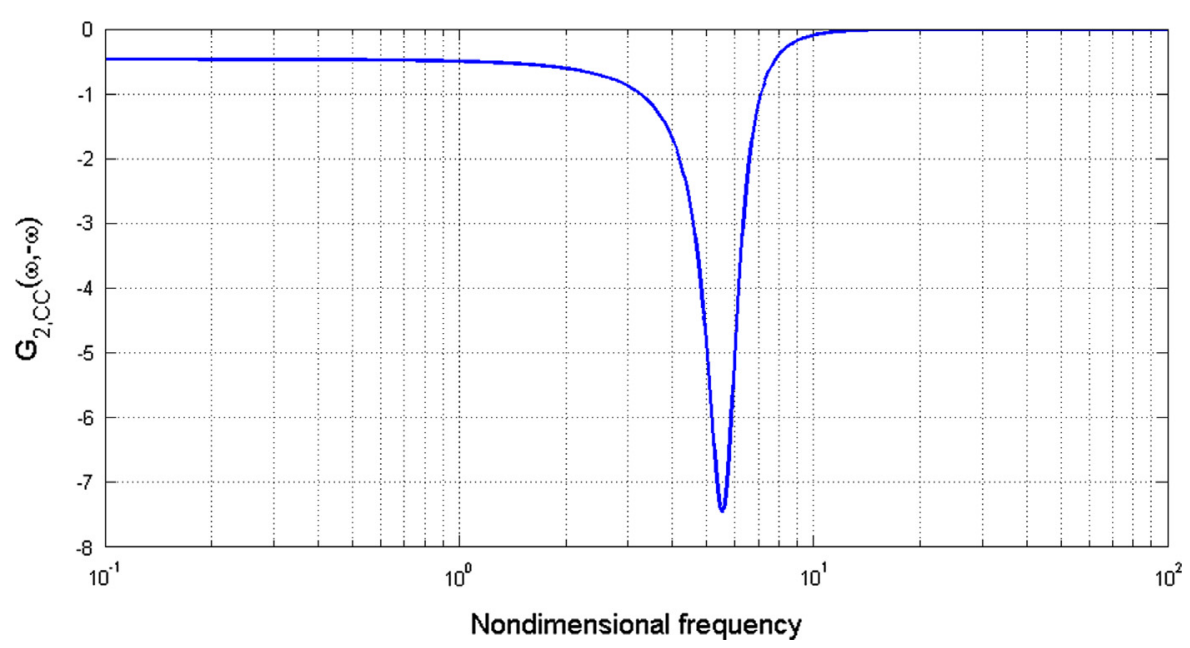

Fig. 5. The second order asymmetrical FRF $G_{2, c c}(\omega,-\omega)$ as a function of frequency (numerical example).

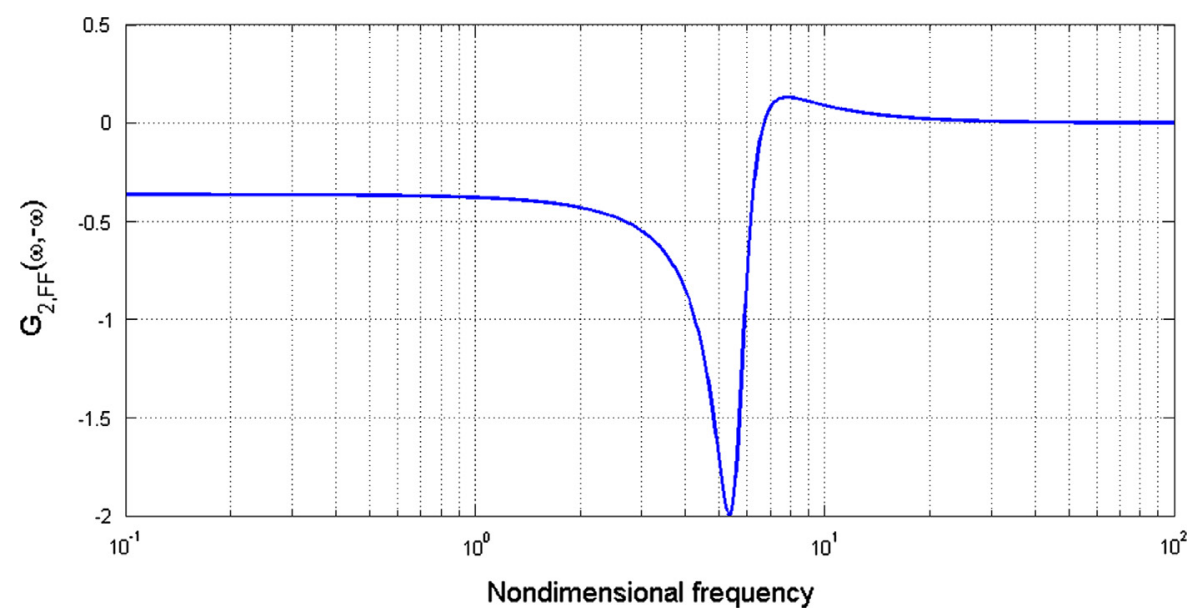

Fig. 6. The second order asymmetrical FRF $G_{2, F F}(\omega,-\omega)$ as a function of frequency (numerical example).
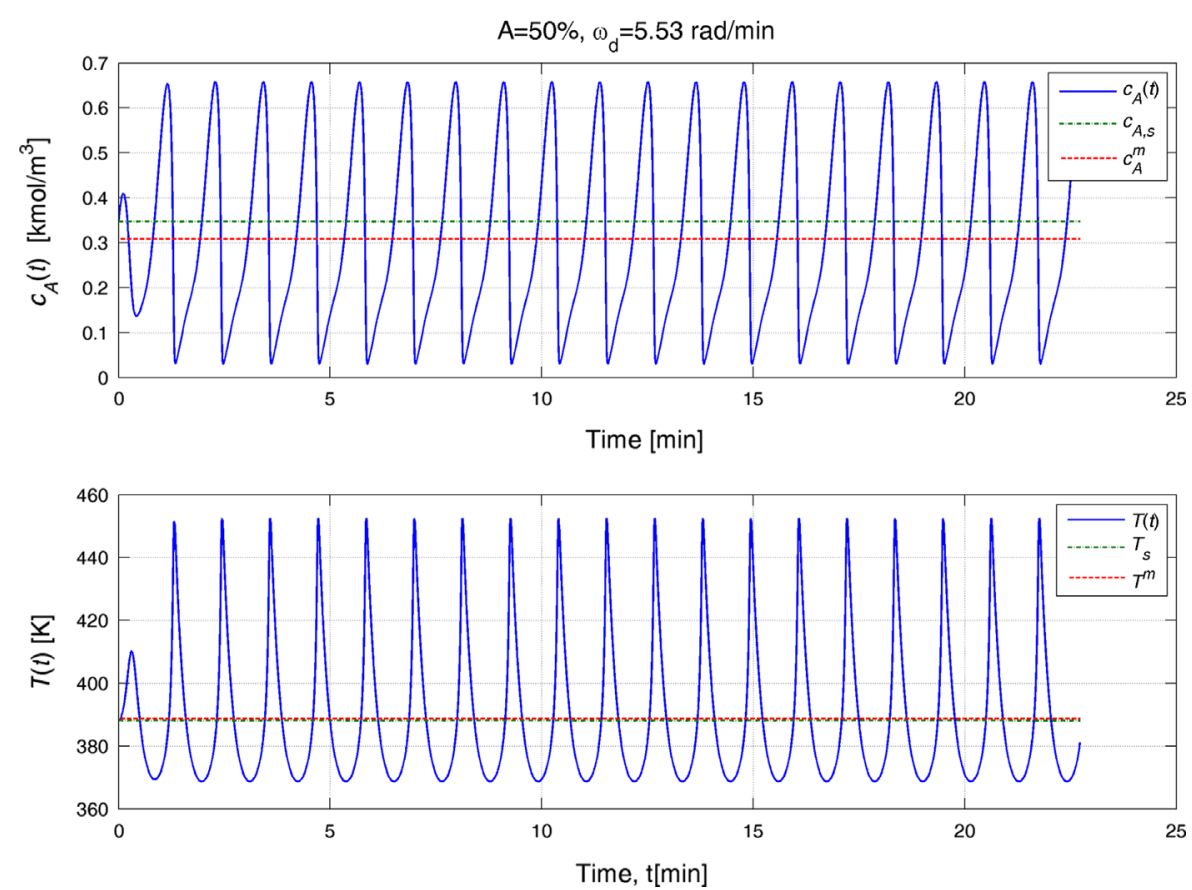

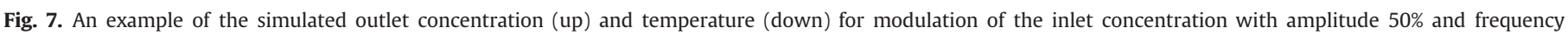
$\omega_{d}=5.53 \mathrm{rad} / \mathrm{min}$. 
Table 5

Concentration change estimated by numerical simulation and by the NFR method.

\begin{tabular}{|c|c|c|c|c|c|c|}
\hline \multirow[t]{3}{*}{ Dimensionless forcing frequency, $\omega$} & \multicolumn{6}{|c|}{ Inlet concentration modulation } \\
\hline & \multicolumn{2}{|c|}{ Input amplitude 50\% } & \multicolumn{2}{|c|}{ Input amplitude $15 \%$} & \multicolumn{2}{|c|}{ Input amplitude 5\% } \\
\hline & $\Delta_{\text {num }}\left[\frac{\mathrm{kmol}}{\mathrm{m}^{3}}\right]$ & $\mathrm{NFR} c_{A, D C}\left[\frac{\mathrm{kmol}}{\mathrm{m}^{3}}\right]$ & $\Delta_{\text {num }}\left[\frac{\mathrm{kmol}}{\mathrm{m}^{3}}\right]$ & $\mathrm{NFR} c_{A, D C}\left[\frac{\mathrm{kmol}}{\mathrm{m}^{3}}\right]$ & $\Delta_{\text {num }}\left[\frac{\mathrm{kmol}}{\mathrm{m}^{3}}\right]$ & NFR $c_{A, D C}\left[\frac{\mathrm{kmol}}{\mathrm{m}^{3}}\right]$ \\
\hline 1 & -0.0296 & -0.0216 & -0.0020 & -0.0019 & -0.00022 & -0.00022 \\
\hline 2 & -0.0313 & -0.0263 & -0.0026 & -0.0024 & -0.00027 & -0.00027 \\
\hline 3 & -0.0315 & -0.0383 & -0.0030 & -0.0034 & -0.00038 & -0.00038 \\
\hline 5 & -0.0368 & -0.2159 & -0.0102 & -0.0194 & -0.0020 & -0.0022 \\
\hline 5.53 & -0.0383 & -0.3237 & -0.0123 & -0.0291 & -0.0027 & -0.0032 \\
\hline 6 & -0.0373 & -0.2203 & -0.0115 & -0.0198 & -0.0021 & -0.0022 \\
\hline 7 & -0.0289 & -0.0504 & -0.0043 & -0.0045 & -0.0005 & -0.0005 \\
\hline 10 & -0.0041 & -0.0041 & -0.0003 & -0.0004 & -0.00004 & -0.00002 \\
\hline \multirow[t]{2}{*}{ Dimensionless forcing frequency, $\omega$} & \multicolumn{6}{|c|}{ Flow-rate modulation } \\
\hline & \multicolumn{2}{|c|}{ Input amplitude 50\% } & \multicolumn{2}{|c|}{ Input amplitude $15 \%$} & \multicolumn{2}{|c|}{ Input amplitude 5\% } \\
\hline 1 & -0.0178 & -0.0164 & -0.0015 & -0.0015 & -0.0002 & -0.0002 \\
\hline 2 & -0.0193 & -0.0186 & -0.0017 & -0.0017 & -0.0002 & -0.0002 \\
\hline 3 & -0.0148 & -0.0238 & -0.0020 & -0.0021 & -0.0002 & -0.0002 \\
\hline 5 & -0.0154 & -0.0747 & -0.0050 & -0.0067 & -0.0007 & -0.0007 \\
\hline 5.53 & -0.0099 & -0.0808 & -0.0043 & -0.0073 & -0.0007 & -0.0008 \\
\hline 6 & -0.0041 & -0.0344 & -0.0021 & -0.0031 & -0.0003 & -0.0003 \\
\hline 7 & 0.0043 & 0.0035 & 0.0003 & 0.0003 & 0.00004 & 0.00004 \\
\hline 10 & 0.0039 & 0.0039 & 0.00036 & 0.00035 & 0.00004 & 0.00004 \\
\hline
\end{tabular}

which indicates the increase of conversion through periodic operation, and its estimate calculated based on the NFR method

$c_{A, D C} \approx c_{A, S}\left(2\left(\frac{A}{2}\right)^{2} G_{2, X X}(\omega,-\omega)\right)$

(where $X X=C C, F F$, for modulation of the inlet concentration or flow-rate, respectively), are given in Table 5 . The results for a number of forcing frequencies, including the resonant one, and for different values of forcing amplitudes, in the range from $5 \%$ to $50 \%$, are given for both modulated inputs.

From the results given in Table 5, we can conclude that

- The conclusions which have been made by the sign analysis could be confirmed by numerical simulations, i.e. increased conversion was achieved in the whole frequency range for inlet concentration modulations, and for forcing frequencies $\omega<\omega_{0, F}=6.71$ for flow-rate modulations. The sign of the predicted concentration change by the NFR method is correct for both inputs and for all forcing frequencies and input amplitudes used for simulation.

- When the input amplitude is kept low (5\%), very good agreements between the approximate (NFR method) and exact (numerical) values of the concentration change are obtained, for both inputs and in the whole frequency range, including the resonant frequency.

- For higher input amplitude (15\%), the agreement between the concentration changes predicted by numerical simulation and the NFR method is still very good, for low and high frequencies, and somewhat worse at and near the resonant frequency. In this frequency range the NFR method overestimates the conversion improvements.

- Finally, for the highest input amplitude (50\%) the results obtained by numerical integration and by NFR method are in a relatively good agreement for low and high forcing frequencies, but differ even for an order of magnitude, for frequencies at and near the resonant one. Again, the NFR method by far overestimates the conversion improvements.

\subsection{Explanation of the largest disagreement observed around the resonant frequency}

The explanation of the disagreement between the results of numerical simulations and the NFR method, for forcing frequencies near the resonant frequency, is due to the fact that the system nonlinearity becomes more pronounced around the resonant frequency (Ritter and Douglas, 1970), and the second order approximation, used in our NFR method (Eq. (4)), is not good enough. In the case of more pronounced nonlinearity, a considerable amount of higher harmonics is expected in the system output In order to investigate the influence of higher order nonlinearities, harmonic analysis of the outlet concentration obtained by numerical simulation was performed, by Fourier analysis.

For illustration, the amplitude spectrum of the outlet concentration, for the case of inlet concentration modulation, with a forcing frequency equal the resonant frequency $\left(\omega_{r}=5.53\right.$ $((\mathrm{rad}) /(\mathrm{min})))$ and high forcing amplitude $(A=50 \%)$ (the data presented in Fig. 7) is graphically presented in Fig. 8. For comparison, the amplitude spectrum obtained with the same forcing frequency, but with low amplitude $(A=5 \%)$ is also presented in Fig. 9.

From Fig. 8, it is evident that for the resonant frequency and high forcing amplitude, the output exhibits a considerable amount of higher harmonics with large gains, which means that the non-linearities of the order higher than two should not be neglected. In the DC component which is of our interest, these higher nonlinearities are defined by the FRFs $G_{4}(\omega, \omega,-\omega,-\omega)$, $G_{6}(\omega, \omega, \omega,-\omega,-\omega,-\omega), \ldots$ (Eq. (3)), which have been neglected in our approximation of the DC component (Eq. (4)). As a consequence, the disagreement between the NFR method and numerical integration is significant. Therefore, in order to evaluate the 


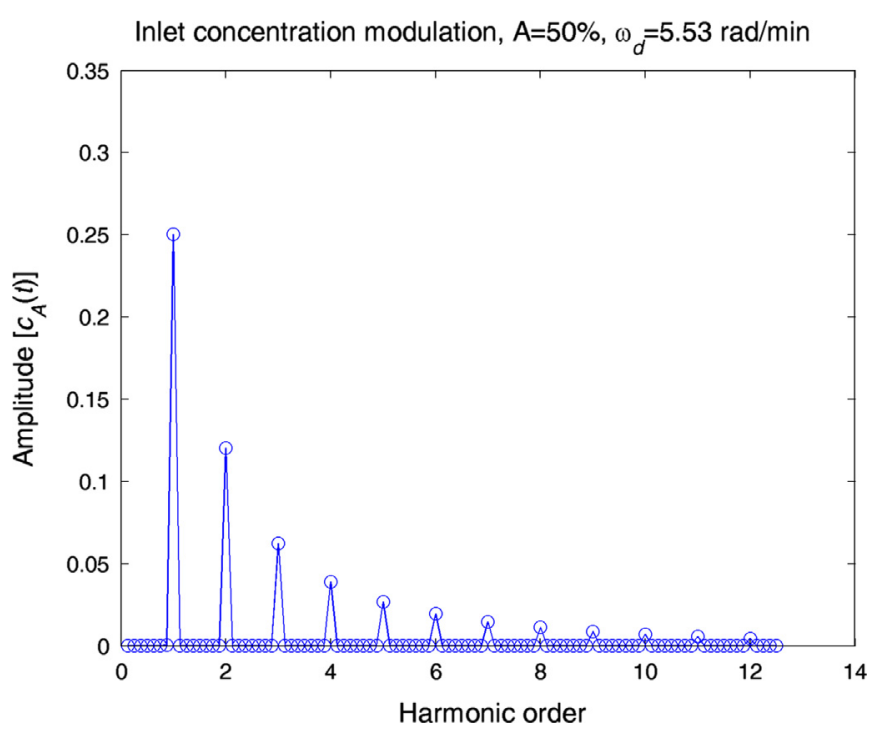

Fig. 8. Amplitude spectrum of the outlet concentration when the inlet concentration is periodically modulated in co-sinusoidal way, with forcing amplitude $A=50 \%$ and forcing frequency $\omega_{d}=5.53 \mathrm{rad} / \mathrm{min}$.

average output concentration for high amplitudes near the resonant frequency more accurately, for the system which exhibit resonance, it would be necessary to derive the higher order FRFs and take them into account.

For the resonant frequency and low forcing amplitude (5\%), the harmonics of order higher than 3 are negligible small (Fig. 9), so in this case Eq. (4), which takes into account only the contribution of second order FRF in the DC component gives a good estimate of the concentration change.

Harmonic analysis of the numerical results obtained for flowrate modulation give very similar results and the same conclusions can be drawn.

\section{Conclusions}

The nonlinear frequency response method is used for evaluation of the possible improvement of a non-isothermal CSTR with simple homogeneous $n$-th order reaction when inlet concentration or flow-rate is periodically modulated. The results presented in this material can be summarized as follows

- The NFR method is applicable only for stable systems, so, before applying it for analysis of periodic operations of a non-isothermal CSTR, it is necessary to analyse the stability of the system.

- Derivation of the asymmetrical second order FRFs for the nonisothermal CSTR is much more complicated than for an isothermal CSTR. The derived FRFs are also much more complex, as is, consequently, the analysis of their sign.

- Contrary to the isothermal CSTR, for which the signs of the $G_{2, C C}(\omega,-\omega)$ and $G_{2, F F}(\omega,-\omega)$ functions depend only on the reaction order (Nikolić-Paunić and Petkovska, 2013), the signs of these functions for the non-isothermal CSTR depend on the reaction order $n$, model parameters $\alpha, \beta, \gamma, \delta$ and St (which depend on the chosen steady-state point) and, in some cases, on the forcing frequency $\omega$.

- When the non-isothermal CSTR is oscillatory stable and the system shows resonant behaviour, the asymmetrical second order FRFs have extrema around the resonant frequency.

- The numerical example shows that the NFR method predicts correctly the sign of the concentration change owing to

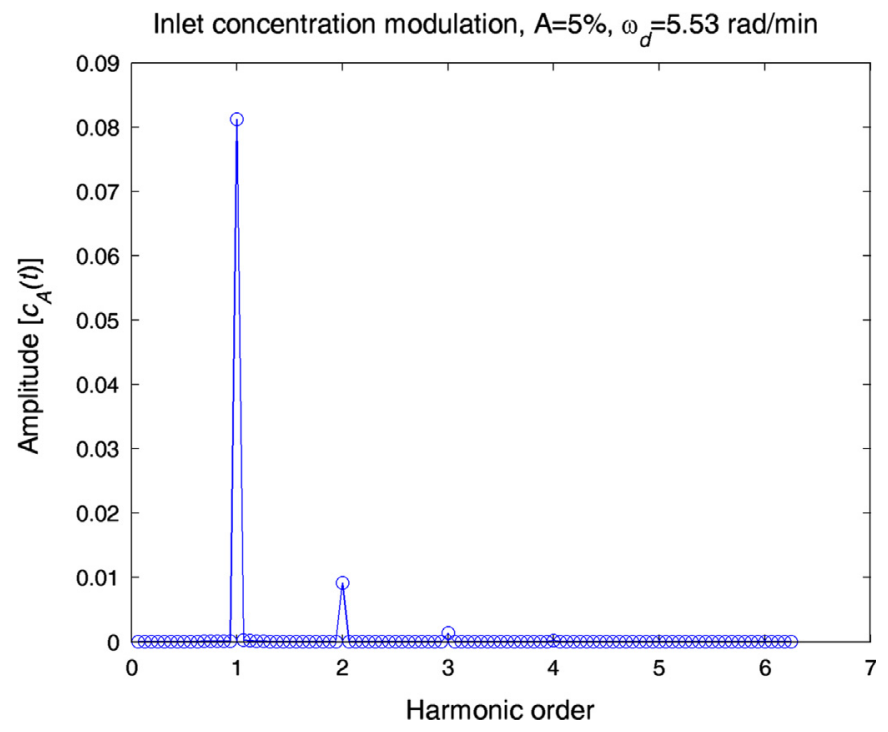

Fig. 9. Amplitude spectrum of the outlet concentration when the inlet concentration is periodically modulated in co-sinusoidal way, with forcing amplitude $A=5 \%$ and forcing frequency $\omega_{d}=5.53 \mathrm{rad} / \mathrm{min}$.

periodic modulation of the inlet concentration or flow-rate, for all frequencies, including the resonant one, and in a wide range of amplitudes.

- Regarding the magnitude of the concentration change owing to periodic modulation of the inlet concentration or flow-rate, the NFR method gives very good predictions in all cases except for high input amplitudes and forcing frequencies at and around the resonant one, where the NFR method highly overestimates the conversion improvement through periodic operation. Wide parameter regions could be identified which provided advantages of forced periodic operation compared to steady state operation. For the particular example case considered, modulating the inlet concentration is more attractive than modulating the feed flow-rate. In the numerical example used in this paper, the steady-state used for comparison was not optimized, so the results presented here cannot be used for drawing conclusions about the optimal periodic vs. optimal steady-state operation.

- The discrepancies between the NFR method and numerical simulation that occurred around the resonant frequencies when high input amplitudes were used, were explained by the extensive nonlinearity, which was proved by harmonic analysis of the numerically simulated output, and cannot properly be approximated by using only the second order asymmetrical FRFs. Including the fourth, and possibly higher order asymmetrical FRFs in the approximation of the DC component is foreseen as a solution for this problem. This issue will be analysed in one of our future publications.

In the second part of this paper (Nikolić et al., 2014) we will apply the nonlinear FR method for analysis of forced periodic operations of a non-isothermal CSTR with modulation of the temperature of the feed stream or the temperature of the cooling/heating fluid.

Finally, we need to point out that, although in this and our previous applications we used the NFR method to predict whether reactant conversion can be increased through periodic operation, in principle the method can also be used to predict possible improvements regarding product selectivity. When conversion is of interest, the FRFs that correlate the outlet reactant concentration and the input modulation are derived and analysed. If, however, the product selectivity is of interest, the FRFs relating the outlet product concentrations to the modulated input need to 
be derived and analysed. Also, although most of our applications have been developed for a CSTR, which is a lumped parameter system, the NFR method is, in principle, also applicable to distributed parameter systems. This was shown in (Marković et al., 2008), where we applied the NFR method to analysis of periodic operations of plug-flow and dispersed plug-flow reactors. We need to point out that in the case of distributed parameter systems the final FRFs are obtained by solving sets of linear ODEs which are obtained after transforming the equations into the frequency domain. This can be much more difficult that solving sets of algebraic equations, which are obtained for lumped parameter systems.

\section{Nomenclature}

A input amplitude

$A_{w} \quad$ surface area for heat exchange

$A_{p s} \quad$ stability parameter

$B \quad$ output amplitude

$B_{p s} \quad$ stability parameter

$c_{A} \quad$ concentration of reactant $\mathrm{A}$

$c_{p} \quad$ specific heat capacity

$C$ dimensionless concentration of reactant A

$E_{A} \quad$ activation energy

$F \quad$ volumetric flow-rate

$F_{n} \quad n$-th order frequency response function which correlates the outlet temperature with the modulated input $n$-th order frequency response function, general and which correlates the outlet concentration with the modulated input

$k_{0} \quad$ preexponential factor in Arrenius equation

reaction order

roots of characteristic equation

reaction rate

universal gas constant

Laplace complex variable

Stanton number

time

temperature

overall heat transfer coefficient

volume of the reactor

input

dimensionless input

output

Greek symbols

$\alpha \quad$ auxiliary parameter

$\beta \quad$ auxiliary parameter

density

dimensionless time

frequency, general and dimensionless

dimensional frequency

resonant frequency

natural frequency

difference between the time-average and the steadystate concentration

difference between the time-average and the steadystate temperature

dimensionless flow-rate
$\Lambda \quad$ auxiliary function
$\Omega \quad$ auxiliary function
$\Delta H_{R} \quad$ heat of reaction

\section{Subscripts}

$\begin{array}{ll}C, C C & \text { inlet concentration modulation } \\ D C & \text { non-periodic term } \\ F, F F & \text { flow-rate modulation } \\ i & \text { inlet } \\ J & \text { heating/cooling fluid (jacket) } \\ \text { num } & \text { numerical } \\ S & \text { steady-state } \\ I & \text { first harmonic } \\ \text { II } & \text { second harmonic }\end{array}$

Superscripts

$m \quad$ mean

Abbreviations

CSTR continuous stirred tank reactor

FR frequency response

FRF frequency response functions

NFR nonlinear frequency response

\section{Acknowledgement}

This work was supported by the Serbian Ministry of Science in the frame of Projects nos. 172022 and III45001.

\section{Appendix A}

A. Taylor series expansions of the nonlinear terms in the dimensionless balance equations (Eqs. (12) and (13))

$$
\begin{gathered}
e^{-\left(E_{A} /\left(R T_{s}(\theta+1)\right)\right)}=e^{-\left(E_{A} /\left(R T_{s}\right)\right)}+\theta \frac{E_{A}}{R T_{S}} e^{-\left(E_{A} /\left(R T_{s}\right)\right)}+\theta^{2}\left(-\frac{E_{A}}{R T_{S}}\right) e^{-\left(E_{A} /\left(R T_{s}\right)\right)} \\
+\frac{\theta^{2}}{2}\left(-\frac{E_{A}}{R T_{S}}\right)^{2} e^{-\left(E_{A} /\left(R T_{s}\right)\right)}+\ldots \\
e^{-\left(E_{A} /\left(R T_{s}(\theta+1)\right)\right)}=e^{-\gamma}\left(1+\theta \gamma+\left(\frac{\gamma^{2}}{2}-\gamma\right) \theta^{2}+\ldots\right) \\
(1+C)^{n}=1+n C+\frac{1}{2} n(n-1) C^{2}+\ldots \\
(1+C)^{n} e^{-\left(E_{A} /\left(R T_{s}(\theta+1)\right)\right)}=e^{-\gamma}\left(1+\gamma \theta+n C+n \gamma C \theta+\left(\frac{\gamma^{2}}{2}-\gamma\right) \theta^{2}\right. \\
\left.\quad+\frac{1}{2} n(n-1) C^{2}+\ldots\right)
\end{gathered}
$$

\section{Appendix B. Derivation of the considered frequency response functions}

The main points of the derivation procedure for the first and asymmetrical second order FRFs when the inlet concentration or flow-rate are modulated, are given here. In the main body of the manuscript, only the final expressions for the second order asymmetrical FRFs which correlate the outlet concentration with 
the modulated inputs are given. The derivation procedure is based on the dimensionless material and energy balances, in which the nonlinearities have been replaced by their Taylor series expansions (Eqs. (14) and (15)).

Derivation of the FRFs $\boldsymbol{G}_{1, \mathbf{c}}(\boldsymbol{\omega}), \boldsymbol{F}_{1, \mathbf{c}}(\boldsymbol{\omega}), \boldsymbol{G}_{2, \mathbf{c c}}(\boldsymbol{\omega},-\boldsymbol{\omega})$ and $\boldsymbol{F}_{2, \mathbf{c c}}(\boldsymbol{\omega},-\boldsymbol{\omega})$

Step 1: Defining the inlet concentration modulation:

$c_{A i}(t)=c_{A i, s}\left(1+A \cos \left(\omega_{d} t\right)\right)$,

$C_{i}(\tau)=A \cos (\omega \tau)=\frac{A}{2}\left(e^{j \omega \tau}+e^{-j \omega \tau}\right)$

Step 2: Representing the outlet concentration and temperature in the form of Volterra series:

$C(\tau)=\frac{A}{2} G_{1, C}(\omega) e^{j \omega \tau}+\frac{A}{2} G_{1, C}(-\omega) e^{-j \omega \tau}+\ldots+2\left(\frac{A}{2}\right)^{2} G_{2, C C}(\omega,-\omega) e^{0}+\ldots$

$\theta(\tau)=\frac{A}{2} F_{1, C}(\omega) e^{j \omega \tau}+\frac{A}{2} F_{1, C}(-\omega) e^{-j \omega \tau}+\ldots+2\left(\frac{A}{2}\right)^{2} F_{2, C C}(\omega,-\omega) e^{0}+\ldots$

Step 3: Substituting the expressions for the inlet concentration, outlet concentration and outlet temperature, defined with Eqs. (B2)-(B4), into the appropriate model Eqs. (14) and (15).

Step 4: Collecting the terms with $(A / 2) e^{j \omega \tau}$, corresponding to the first order functions and with $(A / 2)^{2} e^{0}$, corresponding to the asymmetrical second order functions, and equating them to zero. The resulting equations for the first order FRFs are

$j \omega G_{1, C}(\omega)=(1+\alpha)-(1+n \alpha) G_{1, C}(\omega)-\alpha \gamma F_{1, C}(\omega)$

$j \omega F_{1, C}(\omega)=0-(1+S t+\beta \gamma) F_{1, C}(\omega)-n \beta G_{1, C}(\omega)$

and for the asymmetrical second order FRFs:

$$
\begin{aligned}
0= & 0-2(1+n \alpha) G_{2, C C}(\omega,-\omega)-2 \alpha \gamma F_{2, C C}(\omega,-\omega) \\
& -\alpha\left\{2\left(\frac{\gamma^{2}}{2}-\gamma\right) F_{1, C}(\omega) F_{1, C}(-\omega)+n \gamma G_{1, C}(\omega) F_{1, C}(-\omega)\right. \\
& +n \gamma F_{1, C}(\omega) G_{1, C}(-\omega) \\
& \left.+n(n-1) G_{1, C}(\omega) G_{1, C}(-\omega)\right\} \\
0= & 0-2(1+S t+\beta \gamma) F_{2, C C}(\omega,-\omega)-2 n \beta G_{2, C C}(\omega,-\omega) \\
& -\beta\left(2\left(\frac{\gamma^{2}}{2}-\gamma\right) F_{1, C}(\omega) F_{1, C}(-\omega)+n \gamma G_{1, C}(\omega) F_{1, C}(-\omega)\right. \\
& \left.+n \gamma G_{1, C}(-\omega) F_{1, C}(\omega)+n(n-1) G_{1, C}(\omega) G_{1, C}(-\omega)\right)
\end{aligned}
$$

Step 5: After solving equations for the first order FRFs Eqs. (B5) and (B6) and for asymmetrical second order FRFs Eqs. (B7) and (B8). The final expressions for these FRFs are:

- The first order FRF which correlates the outlet concentration with the modulated inlet concentration

$$
G_{1, C}(\omega)=\frac{(1+\alpha)(1+S t+\beta \gamma+j \omega)}{-\omega^{2}+j \omega(2+\beta \gamma+S t+n \alpha)+(1+n \alpha+\beta \gamma+n \alpha S t+S t)}
$$

- The first order FRF which correlates the outlet temperature with the modulated inlet concentration

$$
F_{1, C}(\omega)=\frac{-n \beta(1+\alpha)}{-\omega^{2}+j \omega(2+\beta \gamma+S t+n \alpha)+(1+n \alpha+\beta \gamma+n \alpha S t+S t)}
$$

- The second order FRF which correlates the outlet concentration with the modulated inlet concentration

$$
\begin{aligned}
& G_{2, C C}(\omega,-\omega)=-\frac{1}{2} \frac{\alpha(1+S t)}{1+n \alpha+\beta \gamma+n \alpha S t+S t} \\
& \quad \times \frac{(1+\alpha)^{2}\left(n(n-1) \omega^{2}+\left[(1+S t)^{2}-2 \beta^{2} \gamma\right] n^{2}-\left[(1+S t+\beta \gamma)^{2}\right] n\right)}{\left[(1+n \alpha+\beta \gamma+n \alpha S t+S t)-\omega^{2}\right]^{2}+\omega^{2}(2+\beta \gamma+S t+n \alpha)^{2}}
\end{aligned}
$$

- The second order FRF which correlate the outlet temperature with the modulated inlet concentration

$$
\begin{aligned}
& F_{2, C C}(\omega,-\omega)=-\frac{1}{2} \frac{\beta}{1+n \alpha+\beta \gamma+n \alpha S t+S t} \\
& \times \frac{(1+\alpha)^{2}\left(n(n-1) \omega^{2}+\left[(1+S t)^{2}-2 \beta^{2} \gamma\right] n^{2}-\left[(1+S t+\beta \gamma)^{2}\right] n\right)}{\left[(1+n \alpha+\beta \gamma+n \alpha S t+S t)-\omega^{2}\right]^{2}+\omega^{2}(2+\beta \gamma+S t+n \alpha)^{2}}
\end{aligned}
$$

B.2. Derivation of the FRFs and $\mathbf{G}_{1, \boldsymbol{F}}(\boldsymbol{\omega}), \boldsymbol{F}_{1, \boldsymbol{F}}(\boldsymbol{\omega}), \boldsymbol{G}_{2, \boldsymbol{F F}}(\boldsymbol{\omega},-\boldsymbol{\omega})$ and $\boldsymbol{F}_{2}$, FF $(\omega,-\omega)$

Step 1: Defining the flow-rate modulation in cosine way

$F(t)=F_{s}\left(1+A \cos \left(\omega_{d} t\right)\right)$

$\Phi_{i}(\tau)=A \cos (\omega \tau)=\frac{A}{2} e^{j \omega \tau}+\frac{A}{2} e^{-j \omega \tau}$

Step 2: Representing the outlet concentration and outlet temperature in the form of Volterra series

$C(\tau)=\frac{A}{2} G_{1, F}(\omega) e^{j \omega \tau}+\frac{A}{2} G_{1, F}(-\omega) e^{-j \omega \tau}+\ldots+2\left(\frac{A}{2}\right)^{2} G_{2, F F}(\omega,-\omega) e^{0}+\ldots$

$\theta(\tau)=\frac{A}{2} F_{1, F}(\omega) e^{j \omega \tau}+\frac{A}{2} F_{1, F}(-\omega) e^{-j \omega \tau}+\ldots+2\left(\frac{A}{2}\right)^{2} F_{2, F F}(\omega,-\omega) e^{0}+\ldots$

Step 3: Substituting the expressions for the flow-rate, outlet concentration and temperature, defined with Eqs. (B14)-(B16), into the appropriate model Eqs. (14) and (15).

Step 4: Collecting the terms with $(A / 2) e^{j \omega \tau}$, corresponding to the first order functions and with $(A / 2)^{2} e^{0}$, corresponding to the asymmetrical second order functions, and equating them to zero. The resulting equations, for the first order FRFs are

$j \omega G_{1, F}(\omega)=\alpha-(1+n \alpha) G_{1, F}(\omega)-\alpha \gamma F_{1, F}(\omega)$

$j \omega F_{1, F}(\omega)=(\beta+S t-\delta)-n \beta G_{1, F}(\omega)-(1+S t+\beta \gamma) F_{1, F}(\omega)$

and for the asymmetrical second order FRFs:

$$
\begin{aligned}
0 & =0-2(1+n \alpha) G_{2, F F}-2 \alpha \gamma F_{2, F F}(\omega,-\omega)-G_{1, F}(\omega)-G_{1, F}(-\omega) \\
& -\alpha\left(2\left(\frac{\gamma^{2}}{2}-\gamma\right) F_{1, F}(\omega) F_{1, F}(-\omega)+n(n-1) G_{1, F}(\omega) G_{1, F}(-\omega)\right. \\
& \left.+n \gamma G_{1, F}(\omega) F_{1, F}(-\omega)+n \gamma G_{1, F}(-\omega) F_{1, F}(\omega)\right) \\
0 & =0-2 n \beta G_{2, F F}(\omega,-\omega)-2(1+S t+\beta \gamma) F_{2, F F}(\omega,-\omega) \\
& -F_{1, F}(\omega)-F_{1, F}(-\omega)-\beta\left(2\left(\frac{\gamma^{2}}{2}-\gamma\right) F_{1, F}(\omega) F_{1, F}(-\omega)\right. \\
& +n(n-1) G_{1, F}(\omega) G_{1, F}(-\omega)+n \gamma G_{1, F}(\omega) F_{1, F}(-\omega) \\
& \left.+n \gamma G_{1, F}(-\omega) F_{1, F}(\omega)\right)
\end{aligned}
$$


Step 5: After solving the equations for the first order FRFs, Eqs. (B17) and (B18), and for the second order asymmetrical FRFs, Eqs. (B19) and (B20)), the following final expressions are obtained:

- The first order FRF which correlates the outlet concentration with the modulated flow-rate

$$
G_{1, F}(\omega)=\frac{\alpha(1+S t-\gamma S t+\gamma \delta)+j \alpha \omega}{-\omega^{2}+j \omega(2+n \alpha+S t+\gamma \beta)+(1+n \alpha+\beta \gamma+n \alpha S t+S t)}
$$

- The first order FRF which correlates the outlet temperature with the modulated flow-rate

$F_{1, F}(\omega)=\frac{(\beta+S t-\delta+n \alpha(S t-\delta))+j \omega(\beta+S t-\delta)}{-\omega^{2}+j \omega(2+n \alpha+S t+\gamma \beta)+(1+n \alpha+\beta \gamma+n \alpha S t+S t)}$

- The asymmetrical second order FRF which correlates the outlet concentration with the modulated flow-rate

$$
\begin{aligned}
G_{2, F F}(\omega,-\omega)=\frac{1}{2} \frac{1}{n \alpha \beta \gamma-(1+n \alpha)(1+S t+\beta \gamma)} \\
\quad \times \frac{1}{\left(\left(-\omega^{2}+1+n \alpha+\beta \gamma+n \alpha S t+S t\right)^{2}+\omega^{2}(2+n \alpha+S t+\gamma \beta)^{2}\right)} \\
\quad \times\left(2 \alpha ( 1 + S t + \beta \gamma ) ( 1 + S t - \gamma S t + \gamma \delta ) \left(-\omega^{2}+1\right.\right. \\
\quad+\beta \gamma+S t+n \alpha(1+S t)) \\
\quad+2 \alpha \omega^{2}(1+S t+\beta \gamma)(2+S t+\beta \gamma+n \alpha) \\
\quad-2 \alpha \gamma(\beta+S t-\delta+n \alpha(S t-\delta))\left(-\omega^{2}+1+\beta \gamma+S t+n \alpha(1+S t)\right) \\
\quad-2 \alpha \gamma \omega^{2}(\beta+S t-\delta)(2+S t+\gamma \beta+n \alpha) \\
+\alpha \gamma(\gamma-2)(1+S t)(\beta+S t-\delta+n \alpha(S t-\delta))^{2} \\
+\alpha \omega^{2} \gamma(\gamma-2)(1+S t)(\beta+S t-\delta)+n(n-1) \alpha^{3}(1+S t) \\
\quad(1+S t-\gamma S t+\gamma \delta)^{2}+n(n-1) \alpha^{3} \omega^{2}(1+S t) \\
+2 n \alpha^{2} \gamma(1+S t)(1+S t-\gamma S t+\gamma \delta)(\beta+S t-\delta+n \alpha(S t-\delta)) \\
\left.+2 n \alpha^{2} \gamma \omega^{2}(1+S t)(\beta+S t-\delta)\right)
\end{aligned}
$$

- The asymmetrical second order FRF which correlates the outlet temperature with the modulated flow-rate

$$
\begin{aligned}
& F_{2, F F}(\omega,-\omega)=\frac{1}{2} \frac{1}{n \alpha \beta \gamma-(1+n \alpha)(1+S t+\beta \gamma)} \\
& \quad \times \frac{1}{\left(\left(-\omega^{2}+1+n \alpha+\beta \gamma+n \alpha S t+S t\right)^{2}+\omega^{2}(2+n \alpha+S t+\gamma \beta)^{2}\right)} \\
& \quad \times\left(2 ( 1 + n \alpha ) \left((\beta+S t-\delta+n \alpha(S t-\delta))\left(-\omega^{2}+1+\beta \gamma+S t+n \alpha+n \alpha S t\right)\right.\right. \\
& \quad+2(1+n \alpha)\left(\omega^{2}(\beta+S t-\delta)(2+n \alpha+S t+\beta \gamma)\right) \\
& \quad-2 n \alpha \beta(1+S t-\gamma S t+\gamma \delta)\left(-\omega^{2}+1+\beta \gamma+S t+n \alpha+n \alpha S t\right) \\
& \quad-2 n \alpha \beta \omega^{2}(2+S t+\gamma \beta+n \alpha) \\
& +\beta \gamma(\gamma-2)\left((\beta+S t-\delta+n \alpha(S t-\delta))^{2}+\omega^{2}(\beta+S t-\delta)^{2}\right) \\
& +n(n-1) \alpha^{2} \beta\left((1+S t-\gamma S t+\gamma \delta)^{2}+\omega^{2}\right) \\
& \left.\quad+2 n \alpha \beta \gamma\left((1+S t-\gamma S t+\gamma \delta)(\beta+S t-\delta+n \alpha(S t-\delta))+\omega^{2}(\beta+S t-\delta)\right)\right)
\end{aligned}
$$

Chen, C.C., Hwang, C., 1994. Optimal periodic forcing of nonlinear chemical processes for performance improvements. Can. J. Chem. Eng. 71, 672-682.

Dorawala, T.C., Douglas, J.M., 1971. Complex reactions in oscillating reactors. AIChE J. 17, 974-981.

Douglas, J.M., 1967. Periodic reactor operation. Ind. Eng. Chem. Process Des. Dev. 6 , 43-48.

Douglas, J.M., 1972. Process Dynamics and Control. Prentice-Hall, Englewood Cliffs, New Jersey.

Douglas, J.M., Rippin, D.W.T., 1966. Unsteady state process operation. Chem. Eng. Sci. 21, 305-315.

Horn, F.J.M., Lin, R.C., 1967. Periodic processes: a varioational approach. Ind. Eng. Chem. Process Des. Dev. 6, 20-30.

Marković, A., Seidel-Morgenstern, A., Petkovska, M., 2008. Evaluation of the potential of periodically operated reactors based on the second order frequency response functions. Chem. Eng. Res. Des. 86, 682-691.

Marlin, E.T., 2000. Process Control: Designing Processes and Control Systems for Dynamics Performance, 2nd edition McGraw Hill, New York.

Nikolić-Paunić, D., Petkovska, M., 2013. Evaluation of periodic processes with two modulated inputs based on nonlinear frequency response analysis. Case study: CSTR with modulation of the inlet concentration and flow-rate. Chem. Eng. Sci. 104, 208-219.

Nikolić, D., Seidel-Morgenstern, A. and Petkovska M., Nonlinear frequency response analysis of forced periodic operation of non-isothermal CSTR using single input modulations. Part II: Modulation of inlet temperature and temperature of the heating/cooling fluid, submitted for publication to Chemical Engineering Science in 2014.

Petkovska, M., Do, D.D., 1998. Nonlinear frequency response of adsorption systems: isothermal batch and continuous flow adsorber. Chem. Eng. Sci. 53, 3081-3097.

Petkovska, M., 2001. Nonlinear frequency response of non-isothermal adsorption systems. Nonlinear Dyn. 26, 351.

Petkovska, M., Marković, A., 2006. Fast estimation of quasi-steady states of cyclic nonlinear processes based on higher-order frequency response functions. Case study: cyclic operation of an adsorption column. Ind. Eng. Chem. Res. 45, 266-291.

Petkovska, M., Nikolić, D., Marković, A., Seidel-Morgenstern, A., 2010. Fast evaluation of periodic operation of a heterogeneous reactor based on nonlinear frequency response analysis. Chem. Eng. Sci. 65, 3632-3637.

Petkovska, M., Seidel-Morgenstern, A. 2013. Evaluation of periodic processes. In: Silveston, R.R., Hudgins, R.R. (Eds.), Periodic Operation of Reactors. Elsevier, Amsterdam, pp. 387-413.

Ritter, A.B., Douglas, J.M., 1970. Frequency response of nonlinear systems. Ind. Emg. Chem. Fundam. 9, 21-27.

Renken, A., 1972. The use of periodic operation to improve the performance of continuous stirred tank reactors. Chem. Eng. Sci. 27, 1925-1932.

Schadlich, K. Hoffmann, U., Hofmann, H., 1983. Periodical operation of chemical processes and evaluation of conversion improvements. Chem. Eng. Sci. 38, $1375-1384$.

Silveston, P.L., 1987. Periodic operation of chemical reactors - a review of the experimental literatureSadhana 10, 217-246.

Silveston, P.L., 1998. Composition Modulation of Catalytic Reactors. Gordon and Breach Science Publishers, Amsterdam.

Silveston, P.L., Hudgins, R.R., Renken, A., 1995. Periodic operation of catalytic reactors - introduction and overview. Catal. Today 25, 91-112.

Silveston, P.L., Hudgins, R.R., 2004. Periodic temperature forcing of catalytic reactions. Chem. Eng. Sci. 59, 4043-4053.

Sterman, L.E., Ydstie, B.E., 1990a. Unsteady-state multivariable analysis of periodically perturbed systems. Chem. Eng. Sci. 45, 737-749.

Sterman, L.E., Ydstie, B.E., 1990b. The steady-state process with periodic perturbations. Chem. Eng. Sci. 45, 721-736.

Sterman, L.E., Ydstie, B.E., 1991. Periodic forcing of the CSTR: an application of the generalized $\Pi$-criterion. AIChE J. 37, 986-996.

Watanabe, N. Onogi, K. Matsubara, M. 1981. Periodic control of continuous stirre tank reactors -I, The pi criterion and its applications to isothermal cases. Chem. Eng. Sci. 36, 809-818.

Weiner, D.D., Spina, J.F., 1980. Sinusoidal Analysis and Modeling of Weakly Nonlinear Circuits. Van Nostrand Reinhold Company, New York.

\section{References}

Bailey, J.E., 1973. Periodic operation of chemical reactors: a review. Chem. Eng. Comm. 1, 111-124. 